ARTICLE

DOI: $10.1038 / \mathrm{s} 41467-017-02413-3$

Corrected: Publisher correction

\title{
Desumoylase SENP6 maintains osteochondroprogenitor homeostasis by suppressing the p53 pathway
}

Jianshuang Li ${ }^{1,2}$, Di Lu${ }^{1}$, Hong Dou ${ }^{3}$, Huadie Liu ${ }^{1,4}$, Kevin Weaver ${ }^{1}$, Wenjun Wang ${ }^{2}$, Jiada Li ${ }^{4}$, Edward T.H. Yeh ${ }^{3}$, Bart O. Williams ${ }^{1}$, Ling Zheng ${ }^{2} \&$ Tao Yang (i) ${ }^{1}$

The development, growth, and renewal of skeletal tissues rely on the function of osteochondroprogenitors (OCPs). Protein sumoylation/desumoylation has emerged as a pivotal mechanism for stem cell/progenitor homeostasis, and excessive sumoylation has been associated with cell senescence and tissue aging, but its role in regulating OCP function is unclear. Here we show that postnatal loss of the desumoylase SUMO1/sentrin-specific peptidase 6 (SENP6) causes premature aging. OCP-specific SENP6 knockout mice exhibit smaller skeletons, with elevated apoptosis and cell senescence in OCPs and chondrocytes. In Senp $6^{-/-}$cells, the two most significantly elevated pathways are p53 signaling and senescence-associated secreted phenotypes (SASP), and Trp53 loss partially rescues the skeletal and cellular phenotypes caused by Senp6 loss. Furthermore, SENP6 interacts with, desumoylates, and stabilizes TRIM28, suppressing p53 activity. Our data reveals a crucial role of the SENP6-p53 axis in maintaining OCP homeostasis during skeletal development.

\footnotetext{
${ }^{1}$ Center for Cancer and Cell Biology, Van Andel Research Institute, Grand Rapids, MI 49503, USA. ${ }^{2}$ Hubei Key Laboratory of Cell Homeostasis, College of Life Sciences, Wuhan University, Wuhan, 430072 Hubei, P. R. China. ${ }^{3}$ Center for Precision Medicine, Department of Medicine, University of Missouri, Columbia, MO 65212, USA. ${ }^{4}$ State Key Laboratory of Medical Genetics and School of Life Sciences, Central South University, Changsha 410078 Hunan, P. R. China. Correspondence and requests for materials should be addressed to T.Y. (email: tao.yang@vai.org) or to L.Z. (email: Izheng@whu.edu.cn)
} 
O steochondroprogenitor cells (OCPs) are derived from the mesenchymal cell lineage and give rise to chondrocytes or osteoblasts. The renewal and maintenance of OCPs are crucial for embryonic skeletal development as well as for postnatal skeletal growth and homeostasis ${ }^{1,2}$. Defective OCP maintenance is considered an important cause of skeletal aging, as the exhaustion of progenitors or stem cells is one of the ultimate culprits of tissue/organism aging ${ }^{3}$.

Emerging evidence indicates that the components of the sumoylation/desumoylation machineries play important roles in stem cell and progenitor maintenance and in tissue aging. For example, the sumoylation E2 enzyme UBC9 is essential for maintaining the survival and pluripotency of embryonic stem cells $(\mathrm{ESCs})^{4}$. SENP2, a SUMO-deconjugating enzyme (desumoylase), sustains the quiescent status of muscle satellite cells and regulates placental trophoblast progenitor expansion and differentiation $^{5,6}$. Overexpression of SUMO2/3 or PIAS $\gamma$ (SUMO E3 ligases) or suppression of the SENP1, 2, or 7 can promote cell senescence ${ }^{7-9}$. In addition, global increases in sumoylation have been detected in tissues derived from aged rats ${ }^{10}$.

The role of the sumoylation/desumoylation processes in bone and cartilage homeostasis has been suggested by several recent studies. UBC9 inhibits bone morphogenetic protein (BMP)induced osteoblast differentiation in cultures partly by enhancing SMAD4 sumoylation ${ }^{11}$, and SENP3 promotes osteogenesis by desumoylating RbBP5 to activate the expression of HOX genes ${ }^{12}$. Moreover, a genomewide association study (GWAS) has shown that a single nucleotide polymorphism (SNP) in the upstream sequence of the SENP6 locus is one of the most significant genetic markers associated with severe osteoarthritis ${ }^{13}$, a disease highly associated with aging. Also, significantly lower SENP6 expression has been found in human osteoarthritic joints ${ }^{14}$.

SENP6 is localized in the nucleoplasm and has a preference for SUMO2/3-modified substrates; it regulates genome stability, cell division, and autoimmune responses, and is involved in adult hematopoietic stem cell renewal ${ }^{15-19}$. In this study, we report that SENP6 is a crucial regulator of the aging process and OCP maintenance during skeletal development. The skeletal phenotype caused by Senp6 loss can be partially rescued by p 53 loss. We further found that SENP6 suppresses p53 activity through interacting with, desumoylating, and stabilizing TRIM28 (tripartite motif-containing 28; also called KAP1 or TIF1 $\beta$ ).

\section{Results}

Loss of Senp6 in adult mice leads to premature aging. Global Senp6 loss in mice causes early embryonic death (data not shown). In order to assess in which postnatal tissues/organs SENP6 has a crucial role, we first bred Senp6 floxed mice (Senp $6 / f)$ with $U B C$ CreERT2 mice (a tamoxifen-induced gene deleter active in all tissues) (Supplementary Figure 1$)^{20}$. Upon tamoxifen injection at 2 months of age, the UBC-CreERT2;Senp $6 / f$ mice progressively developed premature aging complexions, including skin laxity and spotty pigmentation, as well as severe kyphosis at 4 months (Fig. 1a). The kyphosis phenotype implied that SENP6 may play a role in postnatal skeletal maintenance, where mesenchymal stem cells/progenitors have an important contribution ${ }^{21}$. Further, we detected a significant age-related decrease of Senp6 expression in bone marrow stromal cells (BMSCs), a cell population rich in mesenchymal stem cell/progenitors, suggesting that SENP6 levels may be associated with the homeostasis of mesenchymal stem cells/progenitors during aging (Fig. 1b).

Loss of Senp6 in OCPs impairs embryonic skeletal growth. Because stem cell/progenitor exhaustion is one of ultimate hallmarks of aging ${ }^{3}$, and because the sumoylation/desumoylation pathway has been associated with stem cell maintenance and differentiation $^{4-6}$, the aging phenotypes caused by SENP6 loss intrigued us to further assess the specific functions of Senp6 in mesenchymal stem cells/progenitors. We first generated Dermo1Cre;Senp $6^{f / f}$ mice, which had a distinctively small body size and complete penetrance of perinatal death (Supplementary Figure 2). To circumvent the embryonic death, we generated Prx1-Cre; Senp6 $6^{f / f}$ mice, in which the Senp6 gene is specifically deleted in the OCPs of the limb, midline, and a portion of cranial bones, but most of the axial skeleton is not affected ${ }^{22}$. Prx1-Cre;Senp $6^{f / f}$ mice were born at the expected Mendelian ratio. The effectiveness of Cre-mediated gene deletion in these mice was confirmed by qRTPCR in the limb and calvarial tissues, as well as by the GFP expression from the $m$ Tm $G$ allele bred with this line (Prx1-Cre; Senp $\left.6^{f / f} ; m T m G\right)$ (Supplementary Figure 3) ${ }^{23}$.

Prx1-Cre;Senp $6 / f$ mice had severe limb developmental defects at birth, and about $80 \%$ died before weaning, likely due to the difficulty in feeding as a result of their limb defects. Protruding blood sacks on the skulls were noted in the P0 mutant mice, suggesting defective cranial bone development (Fig. 1c). Severe shortening of forelimbs and hindlimbs, abnormally patterned sternums, and distinctively undermineralized skulls were noted in the Prx1-Cre;Senp6 $6^{f / f}$ mice at P0 and P12 (Fig. 1c, d). We also found that P12 Prx1-Cre;Senp $6^{f / f}$ mice had an undermineralized epiphysis (indicated by the lack of alizarin red staining, Supplementary Figure 4), suggesting that these mice had delayed formation of the secondary ossification center (SOC).

Histological staining showed that in the growth plate of P0 Prx1-Cre;Senp6 f/f mice, the lengths of the resting zone (RZ), proliferating zone (PZ), and hypertrophic zone (HZ) were only slightly changed, but the width of the growth plate was dramatically reduced relative to littermate controls (Fig. 1e, Supplementary Figure 5a, b). Moreover, we observed enlarged chondrocytes in the RZ and HZ of the Prx1-Cre;Senp $6^{f / f}$ growth plate (Fig. 1e). The thickness of the perichondrium, which is the OCP-rich tissue surrounding the growth plate and provides new chondrocytes to support growth plate radial expansion, was also markedly reduced (Fig. 1e, Supplementary Figure 5c). The E18.5 Dermo1-Cre;Senp6f/f mice showed growth plate and chondrocyte phenotypes similar to those of Prx1-Cre;Senp6/f mice (Supplementary Figure 6a, b).

Prx1-Cre;Senp $6^{f f f}$ mice showed a severely delayed formation of the SOC, a bony structure developed from the center of the epiphysis separating the articular cartilage from the growth plate ${ }^{1}$. At P12, while the SOCs were clearly established in the proximal femurs of control $\left(\operatorname{Senp} 6^{f / f}\right)$ mice, the epiphysis of the Prx1-Cre; Senp6 f/f mice showed no sign of ossification (Fig. 1f). At P21, when SOC formation was finished in control mice, this process was just initiated in the mutant mice (Fig. 1f). The onset of SOC formation requires the development of cartilage canals, which are invaginated perichondrium invading into the epiphysis center, and they bring OCPs, vessels, and hematopoietic cells for osteogenesis and the establishment of bone marrow ${ }^{24}$. The impaired perichondrium expansion and lack of cartilage canals may contribute to the delay in SOC formation in Prx1-Cre; Senp $6^{f / f}$ mice.

Senp6 loss leads to defective cell homeostasis and DNA repair. Compared with control mice, the growth plate of P0 Prx1-Cre; Senp $6^{f / f}$ mice showed a significantly more TUNEL-positive apoptotic cells, especially in the RZ and periarticular area (Fig. 2a), as well as a significantly fewer BrdU-positive proliferating cells, most notably in the PZ (Fig. 2a). Consistently, total bone marrow from 1-month-old Prx-1-Cre;Senp6 f/f mice showed dramatically fewer colony forming unit-fibroblast 
a

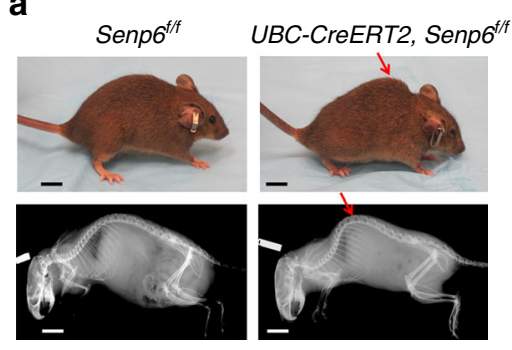

c
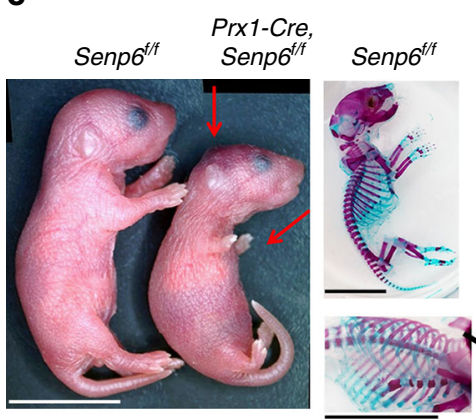

e
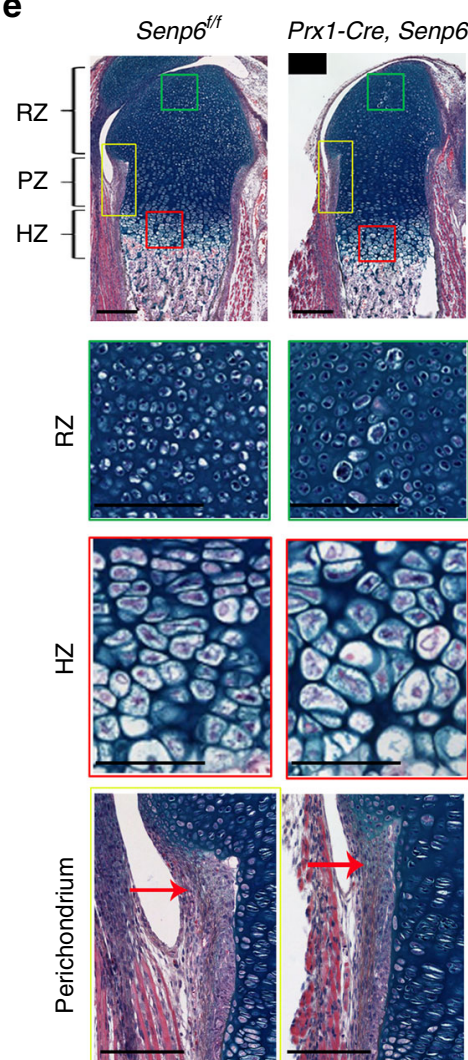

b

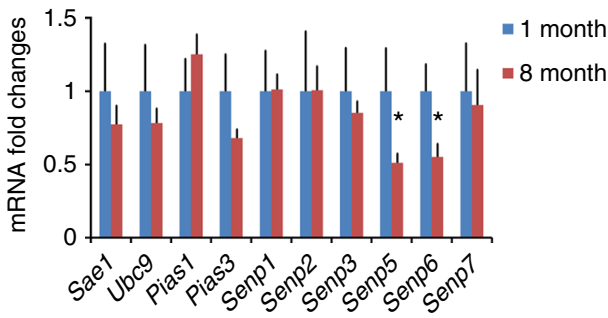

d

Prx1-Cre, Prx1-Cre, $m T m G$, Senp $^{f / t} m T m G$, Senp6 $6^{f / t}$
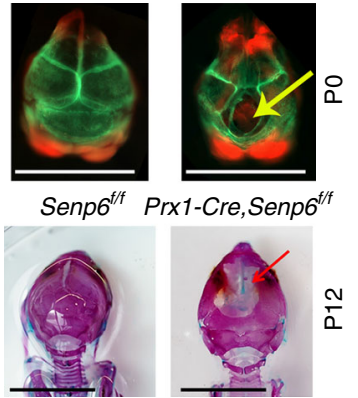

$\frac{N}{2}$

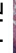

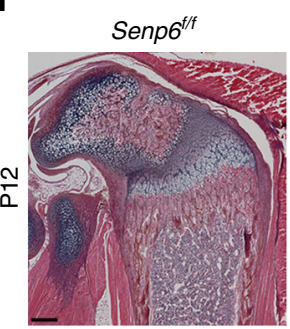

Prx1-Cre, Senp6 $6^{t / f}$
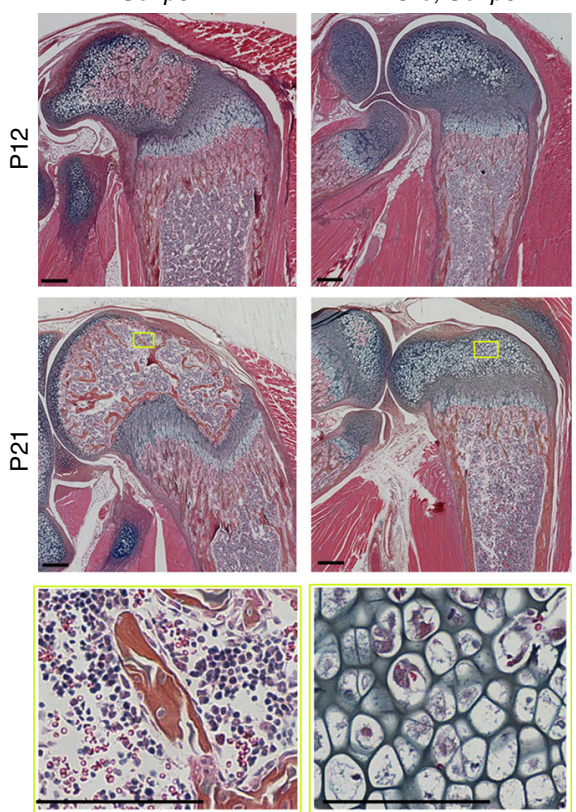

Fig. 1 Senp6 loss caused premature aging and skeletal developmental defects. a Four-momth-old UBC-CreERT2;Senp6t/f mice developed kyphosis (red arrows) after tamoxifen-induced gene deletion (scale bars $=1 \mathrm{~cm}$ ). $\mathbf{b}$ Expression of sumoylation system component genes in BMSCs derived from young ( 1 month) and older ( 8 months) mice $\left(n=4\right.$, error bars $=$ standard deviation, ${ }^{*} p<0.05$, Student's $t$-test). c PO Prx 1 -Cre;Senp6 ${ }^{f / f}$ mice show smaller limbs (red arrows), asymmetric patterning, and decreased ossification of the sternum (black arrow). Scale bars $=1 \mathrm{~cm}$. $\mathbf{d}$ Undermineralized cranial bone in PO Prx1-Cre;Senp6 $6^{f / f} ; m T m G$ mice (upper panel). The green fluorescence indicates the region with active Cre expression; the yellow arrow denotes the opening of the skull. In P12 Prx1-Cre;Senp6 f/f mice (lower panel), the red arrow indicates the Alizarin red-negative region of the skull. Scale bar $=1 \mathrm{~cm}$. e A distal femur of a PO Prx1-Cre;Senp6 $6^{f / f}$ mouse shows a reduced growth plate diameter and enlarged chondrocytes in the resting zone (RZ) and hypertrophic zone $(\mathrm{HZ})$, as well as a thinner perichondrium (red arrows). The colored boxes are enlarged and shown in the lower panels; scale bars $=100 \mu \mathrm{m}$ for the growth plate and $50 \mu \mathrm{m}$ for the rest. f Prx 1-Cre;Senp $6^{f / f}$ mice showed dramatically delayed formation of the secondary ossification center (SOC) at P12 and P21. The yellow boxes are enlarged and shown in the lower panels. Scale bars $=100 \mu \mathrm{m}$ for the growth plate and $50 \mu \mathrm{m}$ for enlarged yellow boxes 
a
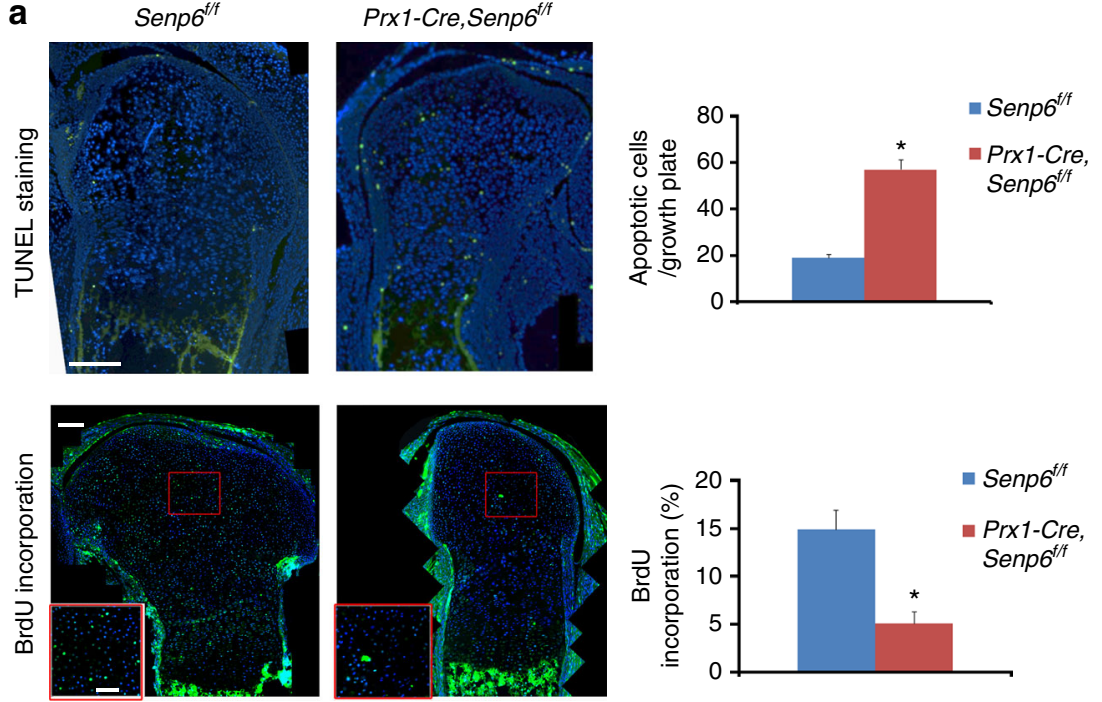

b
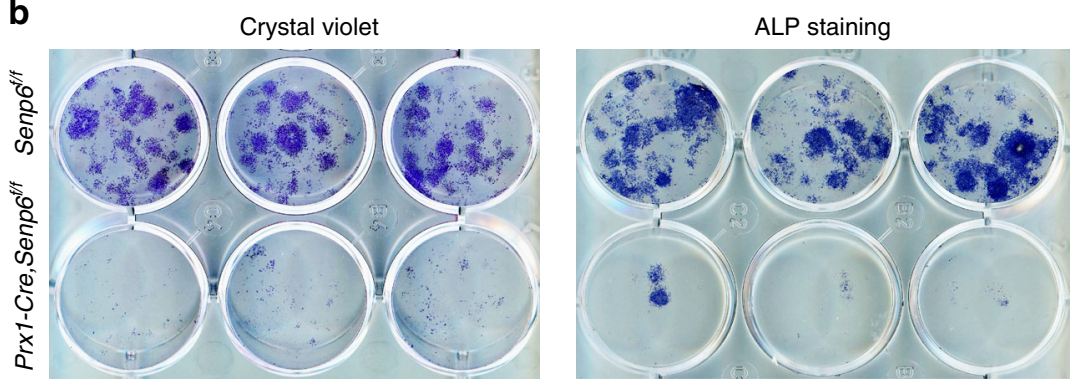

C
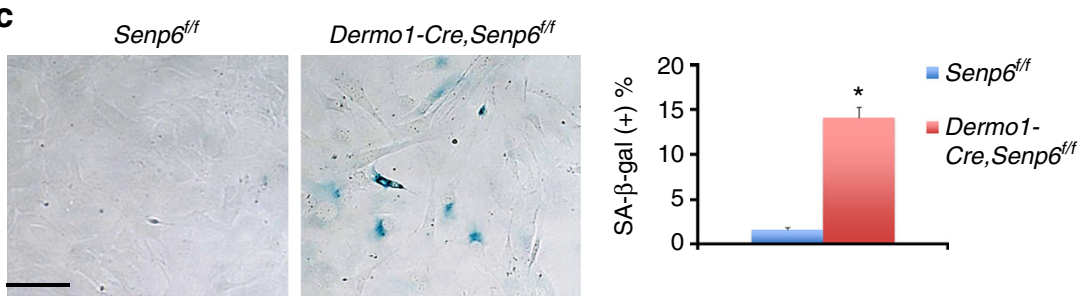

Fig. 2 Dysregulated OCP and chondrocyte homeostasis caused by Senp6 loss. a The growth plate of PO Prx1-Cre;Senp6 $6 / f$ mice showed an enhanced apoptosis (upper panel) and decreased proliferation (lower panel). The red boxed regions were enlarged and are shown in the lower-right corner of each image. Scale bars $=100 \mu \mathrm{m}$ for growth plate and $50 \mu \mathrm{m}$ for the enlarged red boxes. Representative images are on the left and quantifications on the right ( $n=3$, error bars $=$ standard deviation, ${ }^{*} p<0.05$, Student's $t$-test). $\mathbf{b}$ Prx $1-C r e ; S e n p 6^{f / f}$ bone marrow had a decreased number of CFU-F and CFU-O ( $n=3$ ). c Rib chondrocytes cultured from Dermo1-Cre;Senp ${ }^{f / f}$ mouse showed an augmented cell senescence (SA- $\beta$-gal staining); representative images are at the left and quantification at the right. Scale bar $=50 \mu \mathrm{m} ; n=3$, error bars $=$ standard deviation; ${ }^{*} p<0.05$ relative to Senp $6 / f f$ (control), Student's $t$-test

(CFU-F; adhered stromal cells rich in OCPs) and CFU-osteoblast (CFU-O) relative to control (Senp6f/f) mice (Fig. 2b). Similar results were confirmed in the total bone marrow cultures using adenovirus-Cre (Ad-Cre)-mediated Senp6 deletion (Supplementary Figure 7a). Moreover, the Senp6-deleted BMSCs showed a flatter growth curve that reached a plateau earlier than the BMSCs from WT controls (Supplementary Figure 7b). These data collectively suggest that Senp6 loss affected both OCP expansion and OCP proportion in the bone marrow.

The primary rib chondrocyte of early passage (passage 0 ) from Dermo1-Cre;Senp $6^{f / f}$ mice had significantly more senescenceassociated $\beta$-galactosidase (SA- $\beta$-gal)-positive cells than the controls did (Fig. 2c). Increased SA- $\beta$-gal-positive cells were also detected in the growth plate and bone marrow sections of E18.5 Dermo1-Cre;Senp6f/f mice (Supplementary Figure 8). This is consistent with the enlarged chondrocytes observed in the Senp6deficient growth plate, because increased cell volume is one of the signs of cellular senescence. In addition, the expression of another senescence-related marker, $\gamma \mathrm{H} 2 \mathrm{AX}$ (also a DNA damage foci marker), was markedly increased in the mutant growth plates, especially in the RZ, PZ, perichondrium, and periosteum (Fig. 3a). Moreover, X-ray-irradiated Dermo1-Cre;Senp6 $6^{f / f}$ chondrocytes showed dramatically more $\gamma \mathrm{H} 2 \mathrm{AX}$ foci per cell, and the clearance of $\gamma \mathrm{H} 2 \mathrm{AX}$ foci in the recovery phase was significantly delayed (Fig. 3b). It was previously reported that SENP6 knockdown promotes RPA70 sumoylation, which leads to replication stress and DNA breaks ${ }^{15}$. In line with this, we found a marked increase of sumoylated RPA70 in the rib cartilage from E18.5 Dermo1-Cre; Senp $6^{f / f}$ mice (Supplementary Figure 9). These data implied that the unresolved DNA damage, partly caused by RPA70 hypersumoylation, contributed to the elevated cell senescence and apoptosis of SENP6-deficient OCPs.

Senp6 loss activates the $\mathrm{p} 53$ and SASP pathways. To understand the mechanisms underlying Senp6-loss-induced cell senescence 
a

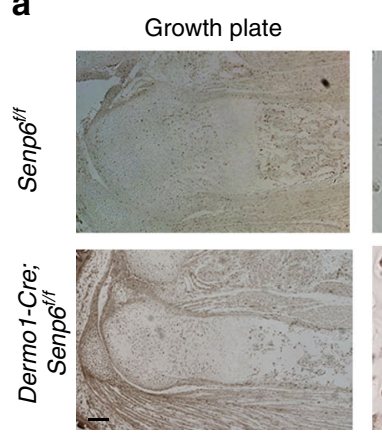

$R Z$
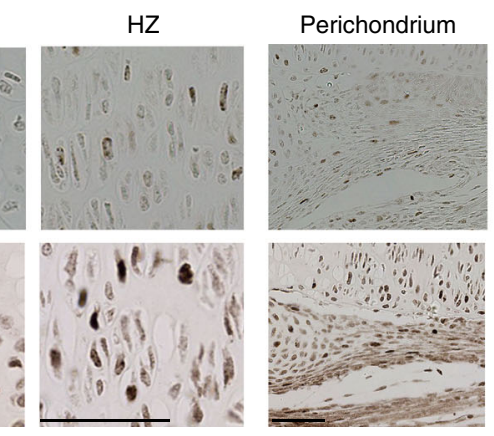

Periosteum
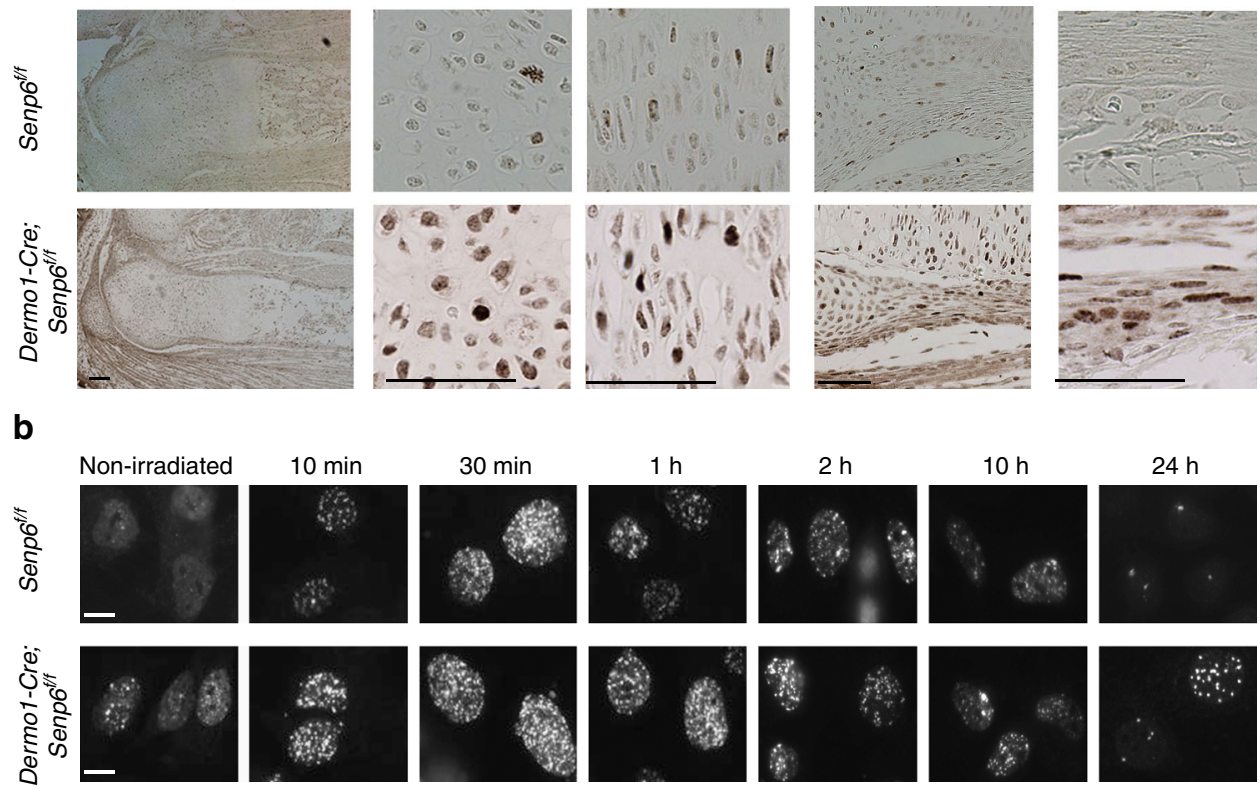

C

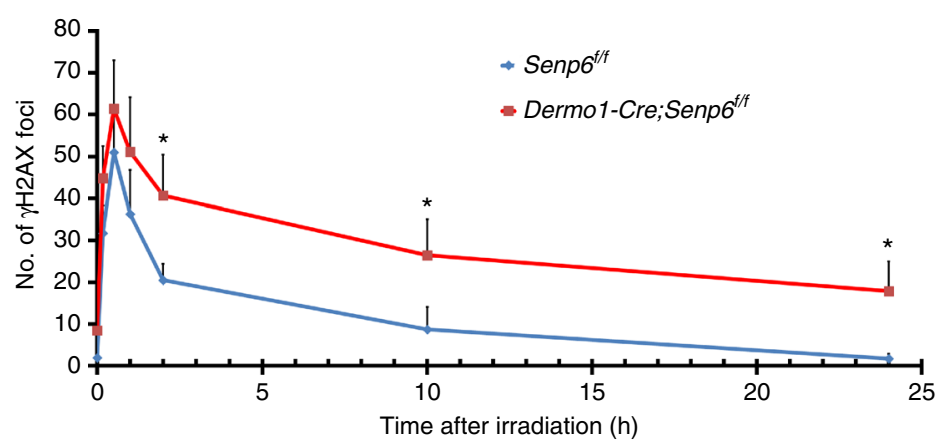

Fig. 3 Senp6 loss delayed DNA damage repair. a Dermo1-Cre;Senp6 ${ }^{f / f}$ mice (E18.5) had more $\gamma \mathrm{H} 2 \mathrm{AX}$-positive cells in the growth plate RZ, PZ, perichondrium, and periosteum than did Senp $6^{f / f}$ controls; scale bars $=100 \mu \mathrm{m}$ for the growth plate (left) and $50 \mu \mathrm{m}$ for the rest. $\mathbf{b}$ Dermol-Cre;Senp6 $f / f$ mice showed an increased basal level of $\gamma \mathrm{H} 2 \mathrm{AX}$ foci (non-irradiated), higher sensitivity to irradiation (at $10 \mathrm{~min}$ ), and lower clearance of $\gamma \mathrm{H} 2 \mathrm{AX}$ foci during recovery at $30 \mathrm{~min}$ to $24 \mathrm{~h}\left(n=3 ; 50\right.$ areas of each slide were quantified, error bars $=$ standard deviation, ${ }^{\star} p<0.05$, relative to control, Student's $t$-test); scale bars $=10 \mu \mathrm{m}$

and apoptosis, we compared the gene expression profiles of cultured rib chondrocytes from Dermo1-Cre;Senp $6^{f / f}$ and Senp $6^{f / f}$ (control) mice by RNA sequencing (RNA-seq). The DAVID Gene Ontology (GO) analysis revealed enriched gene clusters in the Dermo1-Cre;Senp $6 / f$ chondrocytes, including the p53 pathway, the MAPK pathway, and the cytokine-cytokine receptor interaction pathway (Fig. 4a, b). Similar results were obtained from the Gene Set Enrichment Analysis (GSEA) (Fig. 4c). In addition, the SUMO2/3-targeted proteins, total p53, phosphorylated p53 (Ser15), and $\gamma \mathrm{H} 2 \mathrm{AX}$ protein were significantly higher in Dermo1Cre;Senp $6^{f / f}$ samples than in those from control mice (Fig. 4d). However, total SUMO1-modified proteins were not distinctively altered in the Dermo1-Cre;Senp 6/f cartilage (Supplementary Figure 10), in line with the fact that SENP6 has a strong preference for SUMO2/3-modified substrates ${ }^{25}$. Consistent with these data, the expression of $\mathrm{p} 53$ downstream targets that promote apoptosis and senescence (P21, Perp, Pmaip1, and Puma) and senescenceassociated secreted phenotypes (SASP) markers (Ill $\alpha, I l 6, I l 8$, $M m p 3$, and Icam1) were increased, while chondrocyte differentiation and proliferation markers (Sox9, Col2a1, Acan, and Col10a1) were decreased in mutant mice (Fig. 4e). However, in the rib cartilage, Senp6 loss did not affect the expression of other key components of the sumoylation pathway, such as the sumoylation E1, E2, and E3 enzymes and the desumoylases (Supplementary Figure 11). In addition, compared with WT controls, the expression of $C d k n 2 a$, which encodes the p16 protein (an important marker and regulator of cell senescence and aging), had a 1.9-fold increase in the E18.5 Dermo1-Cre;Senp $f^{f / f}$ cartilage (Supplementary Figure 12), although its expression was not significantly changed in the RNA-seq results using cultured primary chondrocytes.

In addition, because p53 overactivity is one of the major mechanisms of organismal aging ${ }^{26}$, these results suggested that the premature aging phenotype seen in the UBC-CreERT2;Senp $6^{f /}$ $f$ mice was likely caused by the hyperactive $\mathrm{p} 53$. In support of this, we found by western blots that the tails from tamoxifen-treated UBC-CreERT2;Senp $6^{f / f}$ mice had a distinctively higher amounts of total p53, p-p53, and SUMO2/3-modified proteins than those from control mice. In addition, the expression of $\mathrm{p} 53$ downstream target genes, such as P21, Perp, Puma, and Pmaip1, was significantly increased, similar to that observed in the Dermo1Cre;Senpofff cartilage (Supplementary Figure 13).

SENP6 suppresses p53 activity by desumoylating TRIM28. Next, we studied how SENP6 regulates the p53 signaling pathway. 
a

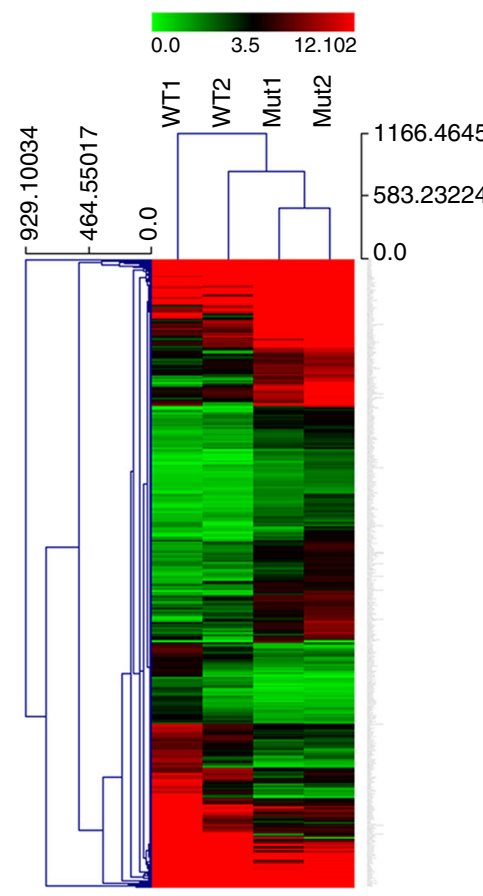

d

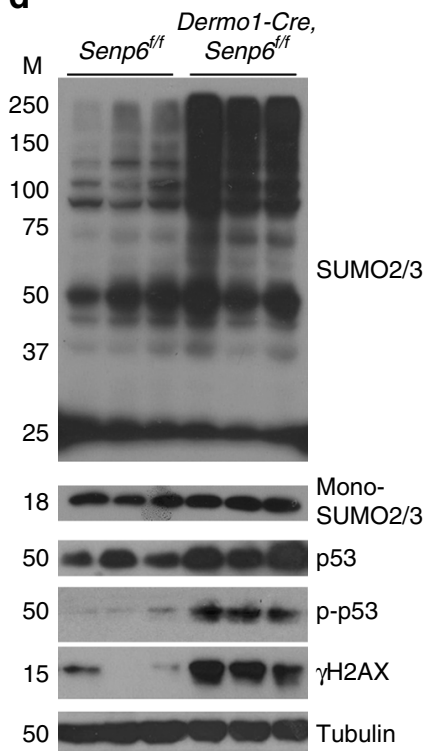

b

\begin{tabular}{|l|c|c|c|c|}
\hline Pathways & $\begin{array}{c}\text { Gene } \\
\text { no. }\end{array}$ & $\%$ & $p$-Value & Benjimini P \\
\hline p53 signaling pathway & 15 & 2.9 & $2.30 \mathrm{E}-08$ & $2.70 \mathrm{E}-06$ \\
\hline MAPK signaling pathway & 19 & 3.7 & $1.80 \mathrm{E}-03$ & $1.00 \mathrm{E}-01$ \\
\hline Cytokine-cytokine receptor interaction & 18 & 3.5 & $1.90 \mathrm{E}-03$ & $7.10 \mathrm{E}-02$ \\
\hline Axon guidance & 12 & 2.3 & $2.90 \mathrm{E}-03$ & $6.70 \mathrm{E}-02$ \\
\hline ECM-receptor interaction & 9 & 1.7 & $4.70 \mathrm{E}-03$ & $8.90 \mathrm{E}-02$ \\
\hline Hypertrophic cardiomyopathy (HCM) & 9 & 1.7 & $5.10 \mathrm{E}-03$ & $8.30 \mathrm{E}-02$ \\
\hline Leukocyte transendothelial migration & 10 & 1.9 & $1.30 \mathrm{E}-02$ & $1.80 \mathrm{E}-01$ \\
\hline
\end{tabular}

C

Enrichment plot: hallmark_P53_pathway
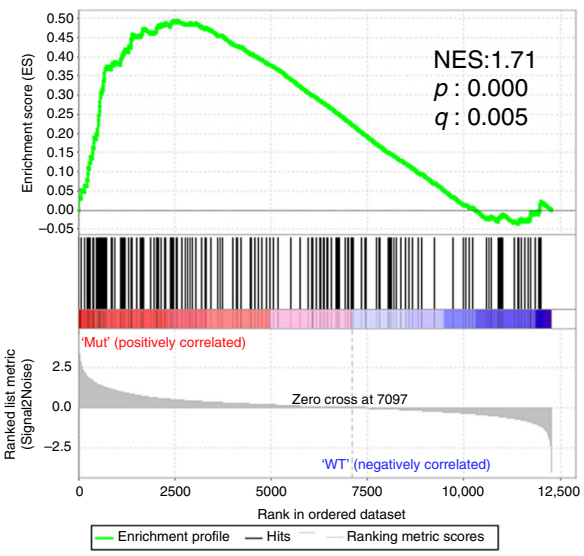

Enrichment plot: hallmark_TNFA_signaling_via_NFKB
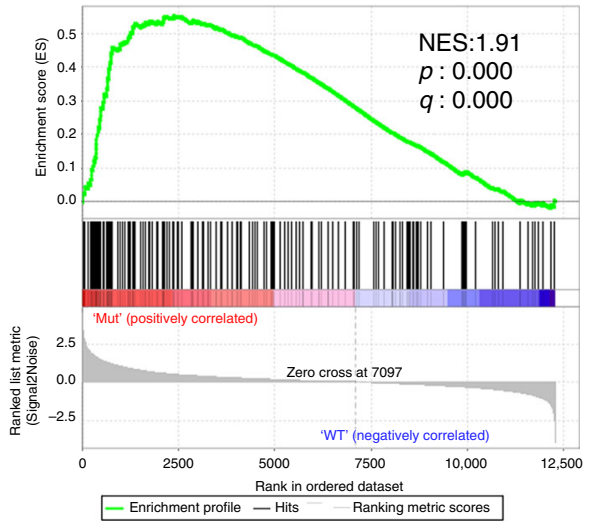

e

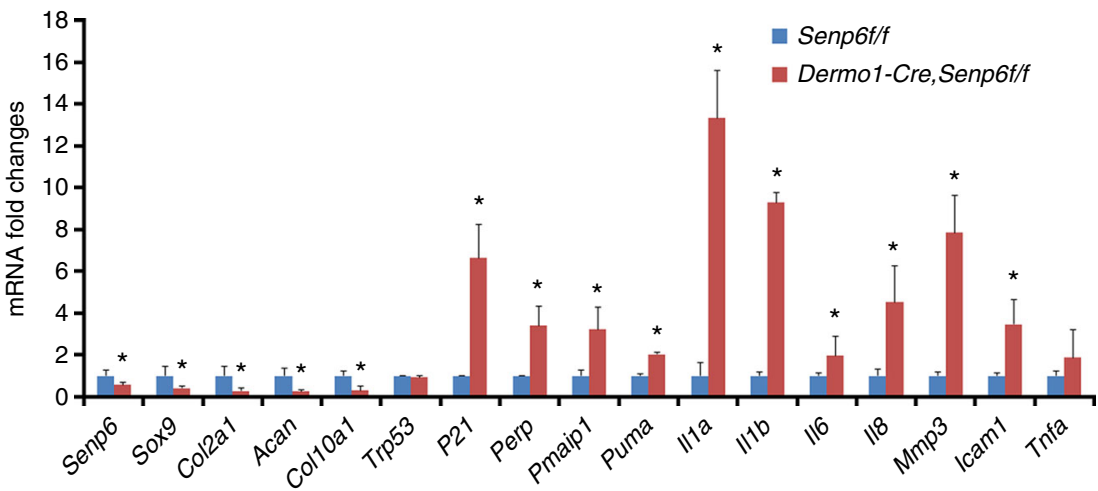

Fig. 4 Senp6 loss activated p53 signaling pathway and SASP. a Heatmap of RNA-seq gene expression profiles comparing Dermo1-Cre;Senp6 $6 / f$ and control primary rib chondrocytes $(n=2)$. b Up-regulated pathways based on DAVID GO analyses ( $p$-values: Fisher Exact/EASE Score). c Enriched p53 pathway and TNF $\alpha$-signaling-via-NFKB pathway in Dermo1-Cre;Senp $6^{f / f}$ by GESA analysis (NES normalized enrichment score; $p$ : nominal $p$-value; $q$ : false discovery rate $q$-value). d Dermo1-Cre;Senp6 $6^{f / f}$ rib cartilage (E18.5) showing higher total SUMO2/3-modified proteins, total and phosphorylated (S15) p53, and $\gamma \mathrm{H} 2 \mathrm{AX}$ relative to controls $(n=3)$. e Chondrocyte differentiation and proliferation markers, p53 downstream targets that promote apoptosis and senescence, and SASP markers were augmented in the rib cartilage of E18.5 Dermo1-Cre;Senp $6^{f / f}$ mice $\left(n=3\right.$; error bars $=$ standard deviation, ${ }^{\star} p<0.05$, relative to controls, Student's t-test)

p53 can be sumoylated at Lys $386^{27}$, but the consequence remains unclear: sumoylation may enhance p53 exportation from the nucleus and degradation, or it may activate p53 by promoting its transactivity ${ }^{28,29}$. In our studies, we were unable to detect sumoylated p53 by western blots using either total cell lysates or a sumoylated protein-enriched fraction from WT and Senp6 $6^{-/}$ chondrocytes (Supplementary Figure 14). Hence, we sought to identify other SENP6 downstream targets that regulate p53 signaling. We enriched the total sumoylated proteins from
Dermo1-Cre;Senp $6^{f / f}$ and Senp6 $f / f$ rib chondrocytes and profiled them using mass spectrometry. Our data showed that TRIM28 was one of the most abundant sumoylated proteins, with 3.2-fold enrichment in Dermo1-Cre;Senp6 ${ }^{f / f}$ rib cartilage (Supplementary Figure 15).

TRIM28 is a multifaceted transcriptional co-repressor involved in chromatin remodeling, stem cell pluripotency, differentiation, DNA damage repair, and p53 regulation ${ }^{30}$. We found more sumoylated TRIM28 (arrow in Fig. 5a) but less total TRIM28 in 
a

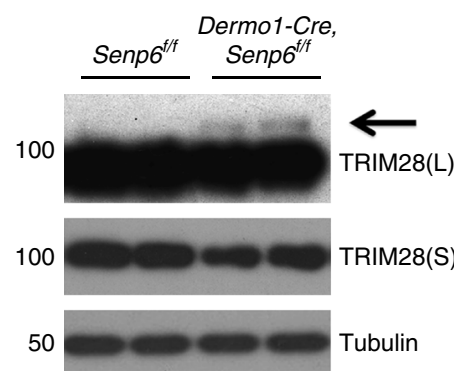

b
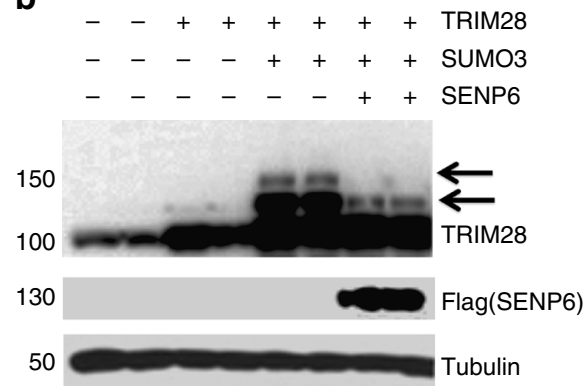

C

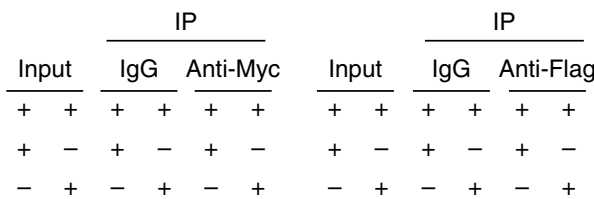

Flag-SENP6

Flag-SENP6(C1030A)

100

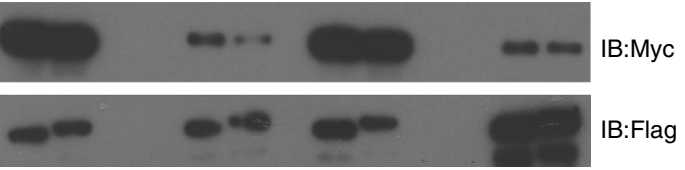

d

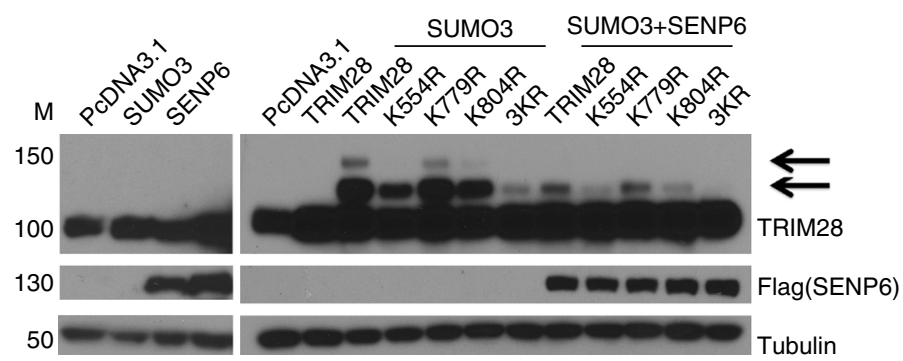

e

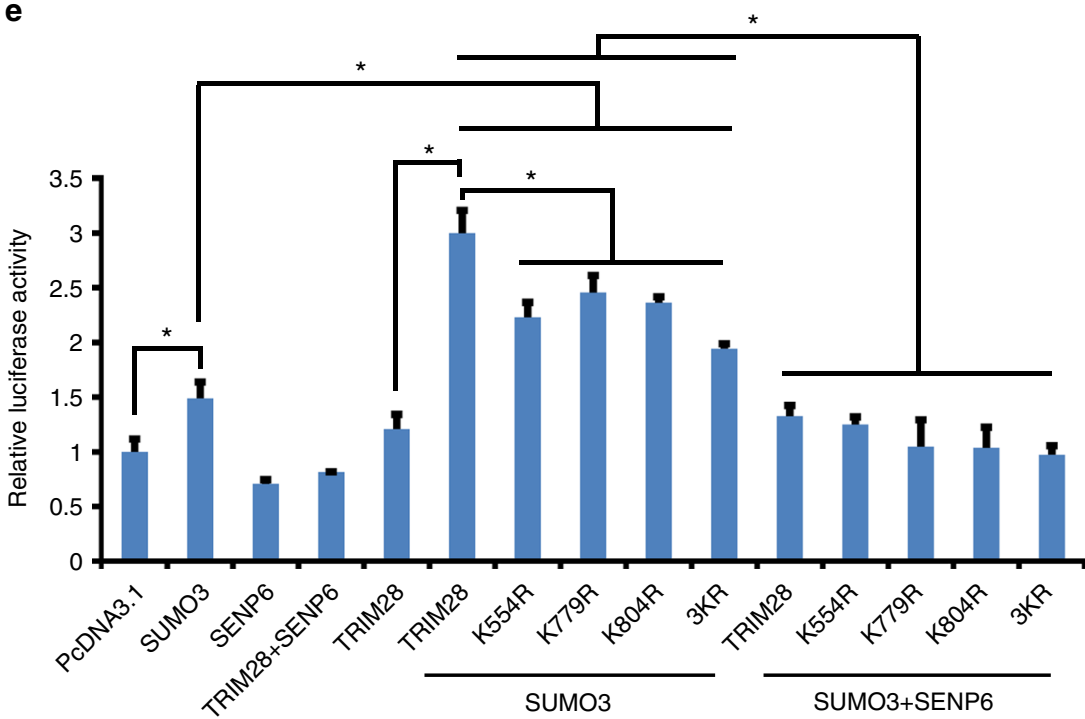

Fig. 5 SENP6 desumoylated and stabilized TRIM28 and suppressed p53. a Senp6 loss decreased the total TRIM28 protein in E18.5 Dermo1-Cre;Senp6 ${ }^{f / f}$ rib cartilage but increased sumoylated TRIM28 (upper bands denoted by the arrow). "L" or "S" indicates long or short exposure ( $n=3$ ). b SUMO3 modifications on TRIM28 can be removed by overexpressed SENP6 $(n=2)$. c TRIM28 can be immunoprecipitated with either wild-type SENP6 or enzymatic-activity-null SENP6 (C1030A). d, e. Sumoylation-resistant TRIM28 mutations impaired TRIM28 sumoylation and p53 activation. Western blots showed that the K554R, K804R, K779R, and 3KR mutations of TRIM28 reduced TRIM28 SUMO3 modifications, and SENP6 effectively removed SUMO3 modification from all these sites (d). p53 reporter luciferase assays showed that the sumoylated TRIM28 enhanced p53 signaling, which was abolished by the addition of SENP6; these TRIM28 mutants induced significantly lower p53 activity than WT TRIM28 did $\left(n=3\right.$; error bars $=$ standard deviation, ${ }^{\star} p<$ 0.05 , Student's t-test) (e) 


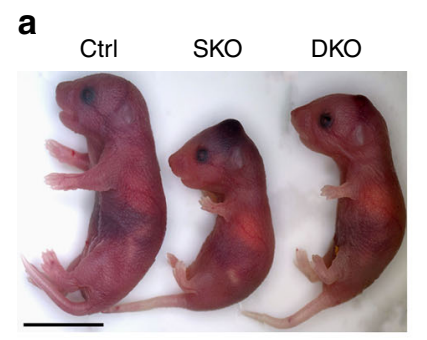

b
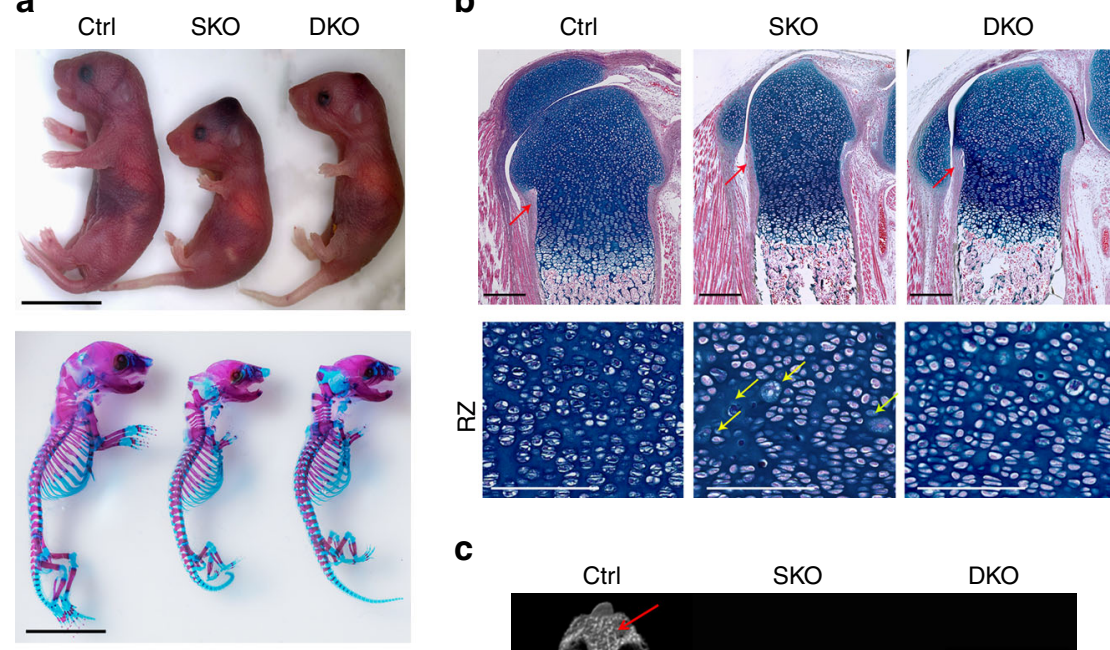

C
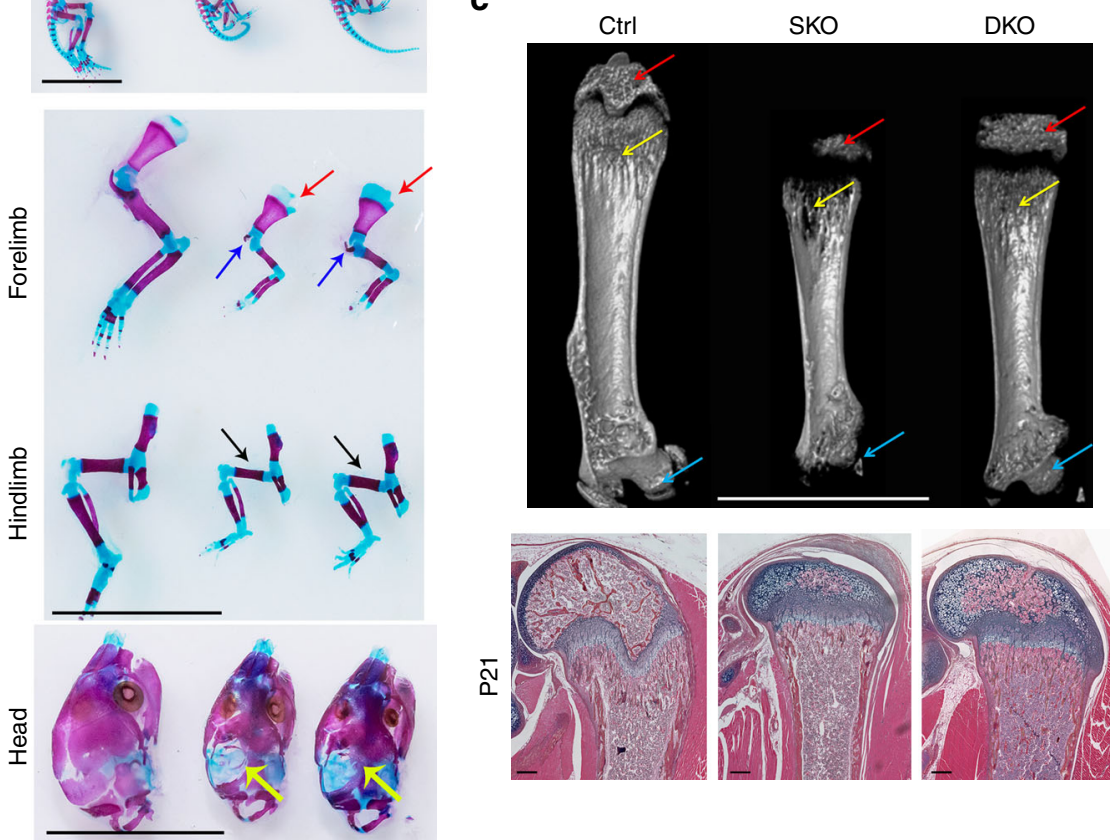

Fig. 6 Trp53 knockout partially rescued skeletal defects caused by Senp6 loss. a Prx1-Cre;Senp6 f/f;Trp53f/f (DKO) PO mice had a partially rescued skeletal phenotype relative to $\operatorname{Prx} 1-C r e ; S e n p 66^{f / f} ; \operatorname{Trp5} 3^{f /+}(\mathrm{SKO})$ mice, as suggested by the smaller indentation in the scapula growth plate (red arrows), the longer clavicles (blue arrows), increased width and length of the femur (black arrows), tibia, humerus, ulna, and radius, and the moderately increased mineralization in the cranial bones (yellow arrows). Scale bars $=1 \mathrm{~cm}$. b PO DKO femoral sections show corrected growth plate diameter, perichondrium thickness (red arrows), and chondrocyte size (yellow arrows) relative to those of SKO mice. Scale bars $=100 \mu \mathrm{m}$ for the upper panels and $50 \mu \mathrm{m}$ for the lower panels. c p53 loss partially corrected the low bone mass and the delayed SOC formation of SKO mice at P21 as revealed by microCT imaging (SOC, red arrows; metaphasis bone, yellow arrows; proximal femur head, blue arrows; scale bars $=1 \mathrm{~cm}$ ). H\&E staining of the corresponding femurs is shown in the lower panels; scale bar $=100 \mu \mathrm{m}$

the Dermo1-Cre;Senp6 $6^{f / f}$ rib cartilage (Fig. 5a). In addition, overexpressed SENP6 effectively removed the SUMO3 modifications on TRIM28 (Fig. 5b). We further found that both WT SENP6 and the catalytic-domain-null SENP6 can be coimmunoprecipitated with TRIM28. These data suggested that a direct SENP6/TRIM28 interaction may mediate TRIM28 desumoylation (Fig. 5c). However, TRIM28 and p53 were not coimmunoprecipitated (Supplementary Figure 16), consistent with a previous report that TRIM28 does not regulate p53 activity by a direct interaction ${ }^{31}$.

Based on published results, TRIM28 has six known sumoylation sites, with three of them crucial to its functions (K554, K779, $\mathrm{K} 804)^{32,33}$. To understand the preference of SENP6 in deconjugating SUMOs from these site(s), we overexpressed in HEK293 cells the TRIM28 variants with point mutations on each of these three sites (TRIM28 $8^{\mathrm{K} 54 \mathrm{R}}$, TRIM28 ${ }^{\mathrm{K} 779 \mathrm{R}}$, TRIM $28^{\mathrm{K} 804 \mathrm{R}}$ ) or with the triple mutation (TRIM28 ${ }^{3 \mathrm{KR}}$ ). Upon SUMO3 overexpression,
TRIM28 $8^{3 \mathrm{KR}}$ showed a strongest resistance to sumoylation (compared with WT TRIM28); TRIM28 ${ }^{\text {K554R }}$ and TRIM28 K804R also had a distinctive decrease, while TRIM $28^{\mathrm{K} 779 \mathrm{R}}$ showed a milder reduction in sumoylation. In addition, the overexpressed SENP6 effectively deconjugated SUMO3 modification from the WT and all these TRIM 28 mutants (Fig. 5d). These data overall suggest that K554, K804, and K779 represented the major sumoylation sites of TRIM28, with the former two most predominant, and that SENP6 was capable of removing SUMO3 modifications from all these three sites.

Next, we evaluated the contribution of TRIM28 sumoylation to the 533 regulation by a reporter luciferase assay. We found that overexpression of SUMO3, TRIM28, or SENP6 alone caused very subtle changes of p53 activity. In contrast, SUMO3/TRIM28 cooverexpression induced a much stronger increase ( $\sim 3$ folds) of p53 activity, and this could be abolished by SENP6 overexpression. In addition, when SUMO3 was overexpressed, all the 
sumoylation-resistant TRIM28 mutants, especially the TRIM28 ${ }^{3 \mathrm{KR}}$, showed less SUMO3 modifications and significantly weaker p53 reporter activity than the WT TRIM28 did
(Fig. 5d, e). These data support the idea that SENP6-mediated TRIM28 desumoylation plays a crucial role in the regulation of p53 activity.

a

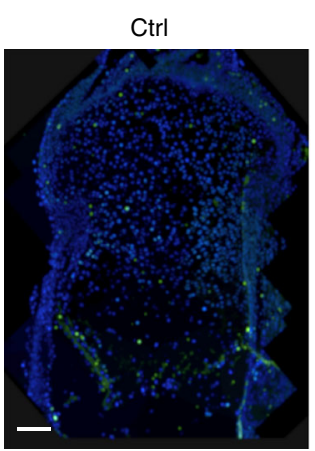

SKO

DKO
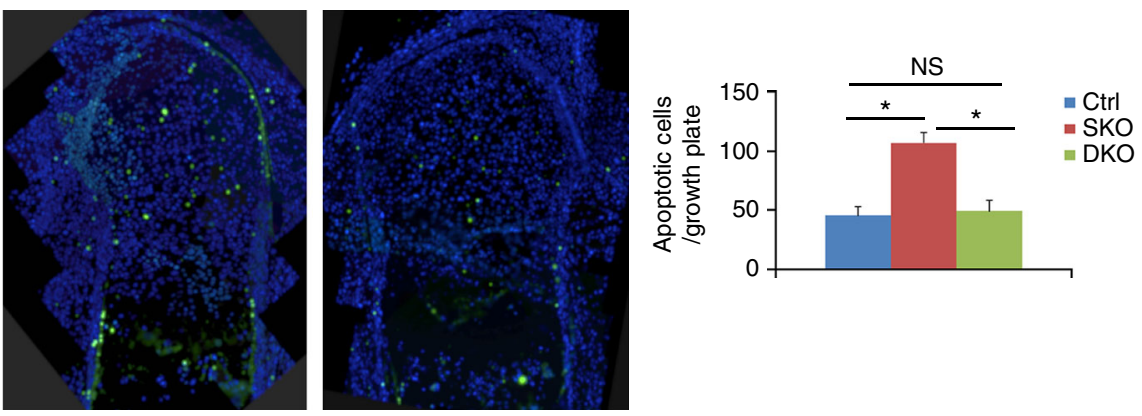

b
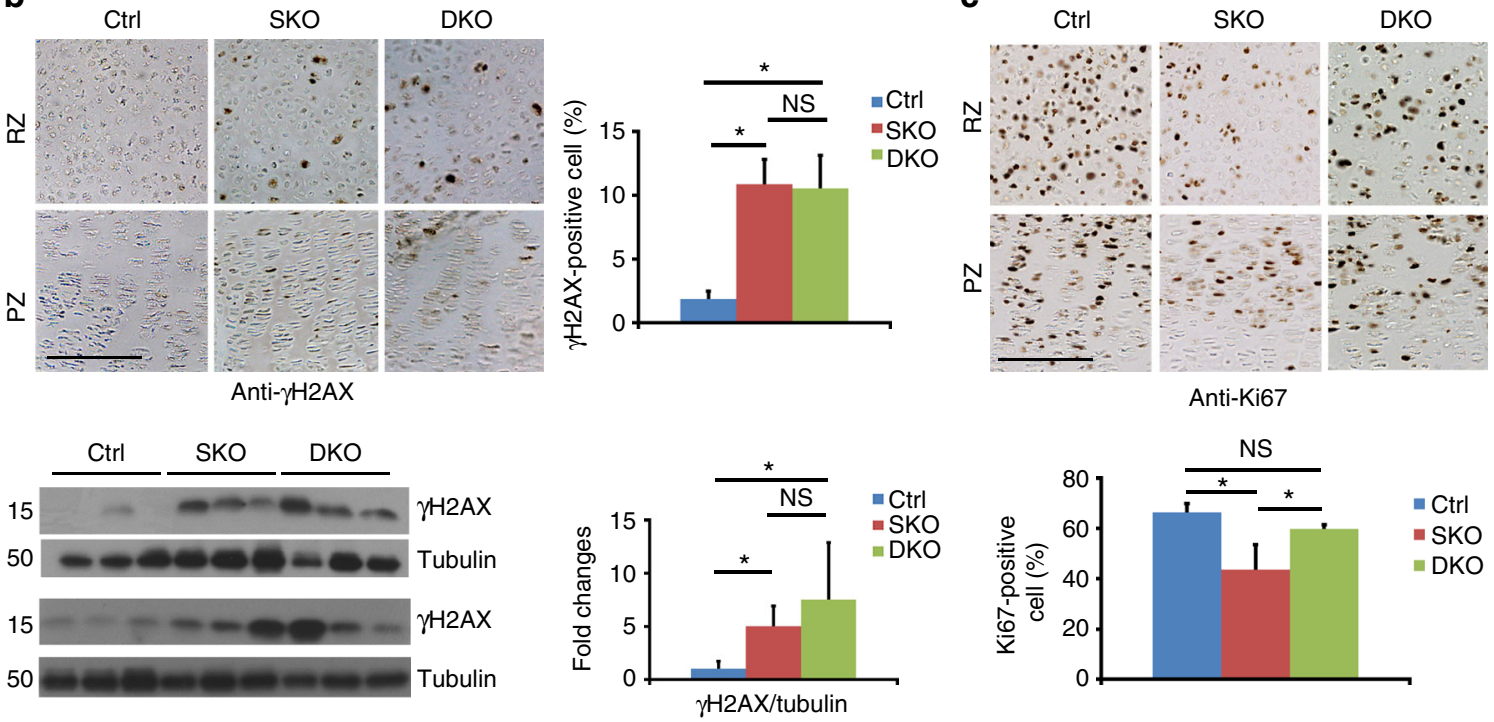

d

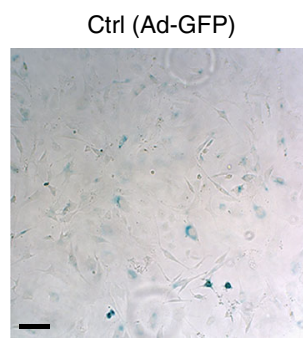

SKO(Ad-Cre)

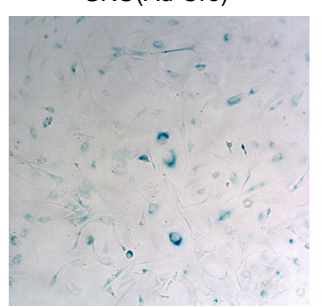

DKO(Ad-Cre)
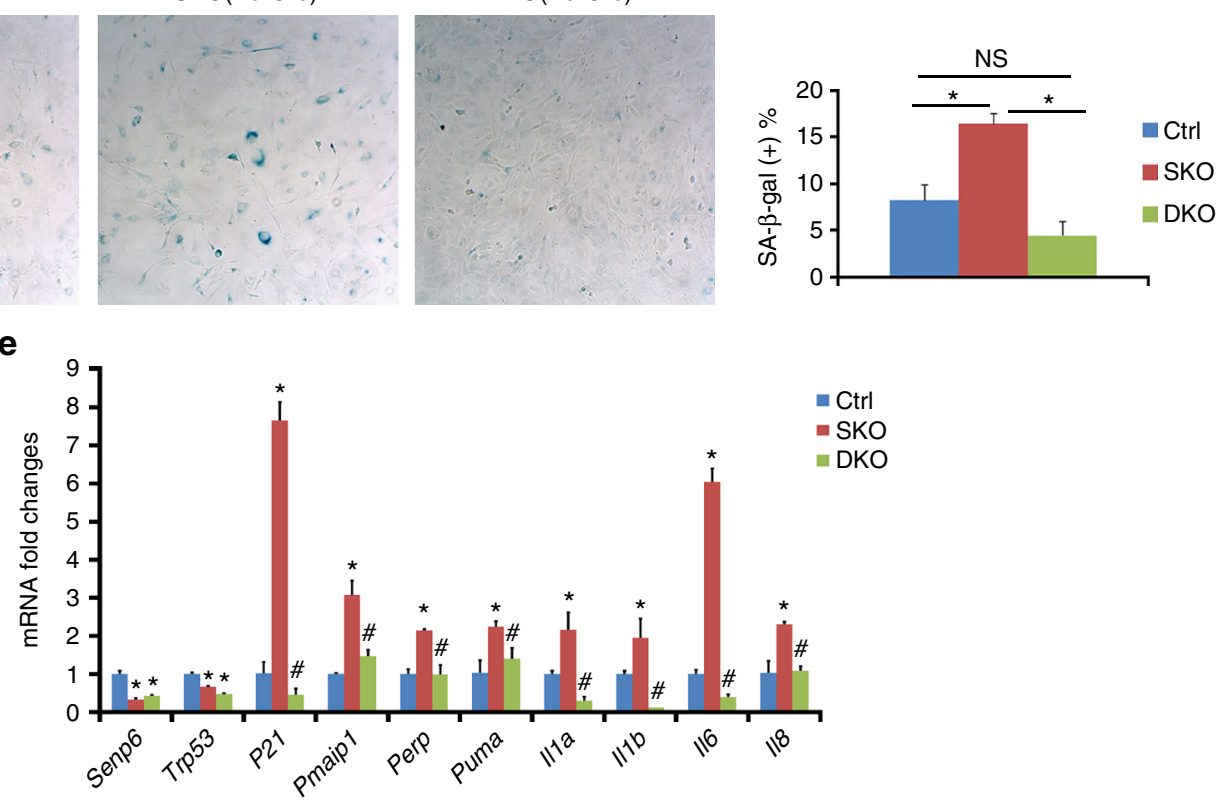
Loss of p53 partially rescues skeletal defects of $\operatorname{Senp6}^{-/-}$mice. To directly test the hypothesis that the SENP6 desumoylase acts through the p53 axis in regulating bone development, we generated Prx1-Cre;Senp6 $6^{f / f} ; \operatorname{Trp} 53^{f / f}$ mice (DKO). Relative to Prx1Cre;Senp $6^{f / f} ; \operatorname{Trp} 53^{f /+}(\mathrm{SKO})$ mice, DKO mice had an improved post-weaning survival rate $(>60 \%)$, increased limb bone length and width, a thicker perichondrium, moderately corrected skeletal morphology and calvarial ossification, and partially rescued chondrocyte size (Fig. 6a, b). Most distinctively, the delayed SOC formation was well corrected (Fig. 6c). DKO mice at P21 showed clear cartilage canal formation in the SOC and a larger ossified area containing bone marrow in the epiphysis center, markedly exceeding those in the SKO mice. In addition, the DKO mice showed a higher trabecular bone mass in both the SOC and the metaphysis of distal femurs than SKO mice did (Fig. 6c). These data collectively suggest that excessive p53 signaling accounts for the skeletal defects in SKO mice.

We then assessed whether $p 53$ deletion could rescue the defect in skeletal cell homeostasis caused by Senp6 loss. Our data showed that DKO mice had significantly fewer apoptotic cells but more proliferating cells in the PZ and RZ of the growth plate. The $\gamma \mathrm{H} 2 \mathrm{AX}$-positive cells in the growth plate of DKO mice were similar to those of SKO mice, and by western blot using growth plate protein samples we detected a moderately but insignificantly higher level of $\gamma \mathrm{H} 2 \mathrm{AX}$ in DKO than in SKO (Fig. 7a, c). DKO primary rib chondrocytes showed less SA- $\beta$-gal staining than did SKO and control chondrocytes (Fig. 7d). Also, the DKO calvarias and femur growth plates had decreased expression of p53 downstream genes and SASP genes (Fig. 7e, Supplementary Figure 17), suggesting that the elevated p53 signaling in Senp6 ${ }^{-/}$ chondrocytes not only promoted apoptosis and cell senescence but also enhanced SASP. This is in line with the previous finding that 553 promotes SASP in the liver cells ${ }^{34}$. Consistently, Senp $6^{-}$ BMSCs were more susceptible to the cell senescence induced by nutlin-3 (a p53 activator) than were control (Senp6 f/f) cells. In contrast, pifithrin- $\alpha$ (PFT $\alpha$, a p53 inhibitor) was more efficient in rescuing the senescence of Senp $6^{-1-}$ BMSCs, relative to Senp6 $6^{f / f}$ BMSCs. This suggests that excessive p53 signaling contributes to the advanced cell senescence of Senp6 $6^{-/-}$cells (Supplementary Figure 18).

\section{Discussion}

Sumoylation regulates the functions of a wide variety of proteins associated with human diseases ${ }^{35}$. However, the role of the sumoylation/desumoylation pathways has not been well studied in skeletal development and aging. In the present work, we found that global deletion of Senp6 in adult mice caused a distinctive premature-aging phenotype. OCP-specific Senp6 deletion resulted in a broad range of skeletal developmental defects associated with enhanced apoptosis and cell senescence. In addition, Senp6 loss markedly elevated inflammatory and tissue-degrading factors of SASP components in the chondrocytes and OCPs. These observations suggest that SENP6 plays a role in preventing skeletal aging by inhibiting both stem cell/progenitor exhaustion and abnormal cell-cell communication, the two ultimate hallmarks of aging $^{3}$. It has been reported that a decreased SENP6 level and an SNP adjacent to the SENP6 locus are associated with osteoarthritis ${ }^{13,14}$, which is triggered and aggravated by articular chondrocyte senescence and the SASP. Whether and how SENP6 plays a role in joint aging and in the pathogenesis of osteoarthritis is worthy of further study.

Our work revealed that Senp6 loss significantly up-regulated p53 signaling. The p53 pathway promotes cell senescence and apoptosis, as well as organismal aging ${ }^{36}$. Mice with a constitutively active p53 mutation (Trp53 ${ }^{T S D}$ ) exhibit severe premature aging with kyphosis ${ }^{37}$, similar to the UBC-CreERT2; Senp $6^{f / f}$ mice. Moreover, a deficiency of MDM2 (an E3 ubiquitin ligase destabilizing p53) in mesenchymal cells or OCPs produces a smaller skeleton and cranial defects ${ }^{38}$, reminiscent of the Dermo1-Cre;Senp6 $6^{f / f}$ or Prx1-Cre;Senp6 f/f mice. We showed that p53 loss can partially but distinctively rescue these Senp6-lossinduced skeletal phenotypes. These results support the idea that the p53 is an important downstream pathway of SENP6 in regulating skeletal development and maintenance. In addition, as a major downstream target of p53, p21 plays a pivotal role in promoting cell senescence. Excessive p21 expression is commonly observed in senescent cells and aging animal models, and the removal of p21 can alleviate cell senescence ${ }^{36}$. We observed a dramatic up-regulation of p21 in Senp6-deficient cells, and further removal of p53 effectively normalized p21 expression and the cell senescence phenotype, implying an important contribution of the p53-p21 axis in Senp6-loss-induced cell senescence phenotype (Fig. 7e, Supplementary Figure 17). Moreover, the wellrescued SOC formation in Prx1-Cre;Senp6 $6^{f / f}$ mice by p53 loss suggested an unprecedented role of p53 signaling in regulating SOC formation, i.e., SOC initiation requires a low local p53 activity.

We showed that SENP6 loss led to increased TRIM28 sumoylation but a decreased total TRIM28 in chondrocytes and OCPs, in line with the findings that TRIM28 is a negative regulator of p53 and cellular senescence. For example, TRIM28 has been shown to promote p53 degradation through MDM2- or MAGEs-dependent pathways $31,39,40$. Also, TRIM28 was reported to be a major target of senescence-associated protein degradation (SAPD), and its loss can trigger cellular senescence in primary fibroblasts ${ }^{41}$. Moreover, sumoylation promotes TRIM28 degradation by RNF4, a sumoylation-dependent ubiquitin E3 ligase $^{42}$. These results suggest that SENP6 may partly prevent p53-hyperactivity-caused cellular senescence by desumoylating and stabilizing TRIM28. Moreover, our luciferase reporter assay showed that the SUMO3 modifications of overexpressed TRIM28 could augment p53 activity, which could be further negated by SENP6 overexpression (Fig. 5e). This suggests that TRIM28 sumoylation could also enhance p53 pathway in a degradationindependent manner. However, these findings apparently differ from previous reports: TRIM28 and sumoylated TRIM28 have been reported to be transcriptional co-repressors of ZBRK1, a zinc-finger DNA-binding protein that mediates transcriptional repression of a few p53 downstream genes ${ }^{43}$; when ZBRK1 is overexpressed, the N-terminal SUMO1-TRIM28 in-frame fusion protein can suppress doxorubicin-induced p21 promoter

Fig. 7 p53 loss rescued the cellular phenotypes of SKO growth plates. a Growth plate of DKO mice shows decreased cell apoptosis, as indicated by TUNEL

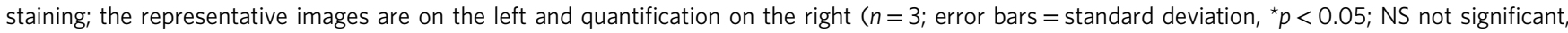
Student's $t$-test; scale bar $=100 \mu \mathrm{m})$. b $\gamma \mathrm{H} 2 \mathrm{AX}$ expression was not rescued in the growth plate of DKO mice. The upper panel shows the representative $\mathrm{IHC}$ on the RZ and PZ of growth plate with quantification on the right; the lower panel shows western blots and quantification on the right ( $n=6$, error bars $=$ standard deviation, ${ }^{\star} p<0.05$, NS not significant, Student's $t$-test; scale bar $=50 \mu \mathrm{m}$ ). c Corrected cell proliferation by Ki67 staining in the growth plate of DKO mice relative to SKO mice (quantification shown in the low panel; $n=3$, error bars $=$ standard deviation, ${ }^{*} p<0.05$, NS not significant, Student's $t$-test; scale bar $=50 \mu \mathrm{m})$. d Corrected cell senescence in Ad-Cre-mediated-DKO vs SKO primary rib chondrocytes, as indicated by SA- $\beta$-gal staining; 
activation in the MCF-7 breast cancer cell line ${ }^{44}$. The discrepancies between these two studies and our result may be due to the reason that our work explored the general role of SUMO3modified TRIM28 sumoylation/desumoylation on p53 activation, while the two studies above focused on ZBRK1-dependent TRIM28 function.

Besides TRIM28, SENP6 has been found to interact with and desumoylate other p53 regulators, such as TIP60 and PML ${ }^{45,46}$. However, PML and TIP60 did not appear in the results of our SUMO IP/MS experiment; their roles in mediating the Senp6loss-induced p53 activation in the mesenchymal cell lineage remain unclear.

We found that Senp $6^{-/-}$chondrocytes had an impaired capacity in clearing irradiation-induced DNA damage. This is consistent with the reports that SENP6 interacts with and desumoylates the RPA70 and Fanconi Anemia ID complex and thus facilitates replication fork processing during DNA repair ${ }^{15,16}$. In support of this, we also found that the level of RPA70 sumoylation was augmented in Senp6-deficient cartilage (Supplementary Figure 9). As a pivotal regulator of DNA damage repair, p53 is quickly activated in response to DNA damage ${ }^{47}$. The dramatically enhanced p53 activity and cell senescence in the Senp6-deficient skeletal cells may be partly due to a prolonged stress response to the impaired DNA repair. However, in HEK293 cells, TRIM28/ SUMO3 co-overexpression did not seem to alter the level of $\gamma \mathrm{H} 2 \mathrm{AX}$ (Supplementary Figure 19), while it was still sufficient to enhance 553 reporter activity (Fig. 5e). This implies that, at least in such an experimental setting, the SENP6-TRIM28 axis could regulate p53 pathway in a DNA damage-independent manner. Interestingly, we found that $p 53$ loss partially rescued the skeletal phenotype and cell senescence caused by Senp6 deficiency, but it did not rescue (or even worsen) the DNA damage. This phenomenon is reminiscent of several previous reports: Trp53 loss can rescue the premature aging and cell senescence phenotypes of Brcal or $K u 80$ knockout mice at the expense of increasing DNA damage $^{48,49}$.

We showed that p53 loss resulted in a distinct but incomplete rescue of Senp6-KO phenotypes, implying that other p53independent pathways may also contribute to the regulation of SENP6-mediated OCP homeostasis. Moreover, TRIM28 and its sumoylated forms have been found to orchestrate chromatin remodeling by modulating DNA and histone modifications ${ }^{50,51}$. In support of this, a recent finding showed that both TRIM28 and SENP6 are important factors in suppressing endogenous retrovirus activation, which is commonly implemented through chromatin silencing ${ }^{52}$. How the SENP6/TRIM28 interaction epigenetically regulates stem cells/progenitor dynamics is a promising direction for further exploration.

Interestingly, SENP7, another member of desumoylase family, has also been found to modulate TRIM28 function in chromatin relaxation, which is an important biological event for DNA damage repair and epigenetic regulation ${ }^{53}$. Our RNA-seq data showed that SENP7 $(\mathrm{RPKM}=4.85)$ was expressed at a relatively lower level than SENP6 $(\mathrm{RPKM}=14.4)$, and its expression was unaltered in the BMSCs of aging mice and unaffected by Senp6 loss in the chondrocytes (Fig. 1b, Supplementary Figure 11). Also, desumoylase SENP5 has been reported to regulate DNA repair and mitochondrial function, both of which are highly associated with aging ${ }^{54,55}$. We found that Senp5 expression was decreased in the BMSCs of aging mice (Fig. 1b), but its expression was not affected by Senp6 loss in the chondrocytes (Supplementary Figure 11). It remains unclear whether SENP7 and SENP5 have a redundant role of SENP6 in the skeletal context.

Overall, our study suggests that during skeletal development, SENP6 desumoylase maintains proper OCP renewal, differentiation, and survival, partly through suppressing p53 pathways.
These findings will shed new light into research in fields such as cell senescence, stem cell rejuvenation, aging, and cancer, where sumoylation and p53 pathways play crucial roles.

\section{Methods}

Mice. The Dermo1-Cre, Prx1-Cre, UBC-CreERT2, and Trp53fff mice were obtained from Jackson Lab and maintained by breeding with WT C57BL/6 strains. The Senp6 floxed mice were created by the laboratory of Dr. Edward Yeh and maintained on C57BL/6 background. Mice at embryonic or perinatal stages were not separated by sexes. The mice at p12, p21, and adult age used in the present study were males. The littermates were randomly grouped based on genotypes for all the experiments. The animal procedures were approved by the Van Andel Research Institute Animal Care and Use Committee.

Skeletal staining. The scarified mice with skin and internal organs removed were fixed in $95 \%$ ethanol overnight and stained with alcian blue $(0.3 \%$ in $70 \%$ ethanol $)$ for $24-48 \mathrm{~h}$. Next, the skeletons were incubated with $2 \% \mathrm{KOH}$ for $24-48 \mathrm{~h}$, then stained with alizarin red S $(0.1 \%$ in $95 \%$ ethanol $)$ overnight. The stained skeletons were cleared in $1 \% \mathrm{KOH} / 20 \%$ glycerol solution for 2-3 days, and stored in ethanol/ glycerol solution $(1: 1)$.

Histology and immunohistochemistry. For histological analyses, embryonic undecalcified or adult decalcified (using 14\% EDTA solution) mouse skeletal tissues were fixed in $4 \%$ paraformaldehyde and were paraffin-embedded. Specimens were sectioned at a thickness of $5 \mu \mathrm{m}$. Sections were stained with Alcian blue hematoxylin/orange $\mathrm{G}$ following standard procedures.

For immunohistochemistry, sections were dewaxed and rehydrated, antigenunmasked in citrate buffer, and then were quenched for endogenous peroxidase activity using $3 \% \mathrm{H}_{2} \mathrm{O}_{2}$ for $10 \mathrm{~min}$. After rinsing in $\mathrm{PBS}$, the sections were incubated in the normal serum blocker (NSB) buffer ( $3 \%$ normal serum, $0.1 \%$ BSA, and $0.1 \%$ Triton $\mathrm{X}-100$ in PBS) for $30 \mathrm{~min}$ and then were reacted with specific primary antibodies overnight at $4{ }^{\circ} \mathrm{C}$. After washing, sections were incubated with biotinylated secondary antibody for $1 \mathrm{~h}$ at room temperature, treated with avidin-biotin-peroxidase complex reagent (ABC, Vector Laboratories) for $30 \mathrm{~min}$ at room temperature, followed by incubation with peroxidase substrate solution (DAB) (Vector Laboratories). Finally, sections were counterstained with hematoxylin for $20 \mathrm{~s}$ and mounted with coverslips ${ }^{56,57}$. Detailed information about the antibodies used in this study is provided in Supplementary Table 1.

microCT imaging. Following the JBMR-recommended microCT guidelines and previous publications ${ }^{58,59}$, we scanned the P21 male mouse femurs at $13.3-\mu \mathrm{m}$ voxel size $(59 \mathrm{kV}, 167 \mu \mathrm{A})$ using a SkyScan 1172 microCT imaging system. After reconstruction using the RNecon software with a threshold set at 90 and aligned in the Dataviewer software, 3D images were created using the CTan software. The sagittal section views of femurs were captured using the CTvol software.

BrdU incorporation and TUNEL assays. For the cell proliferation assay, BrdU labeling reagent ( $10 \mu \mathrm{l} / \mathrm{g}$ body weight; Life Technologies) was injected into pregnant female mice or postnatal mice. Two hours later, the mice were sacrificed, and the growth plate samples were collected for sectioning. The tissue sections were dewaxed and permeabilized with $0.5 \%$ Triton X-100:PBS, blocked in NSB buffer, and stained with anti-BrdU antibody (Life Technologies) and anti-mouse AlexaFluor-488 secondary antibody (Molecular Probes). Mounting medium containing DAPI was used to counterstain nuclei. Images were acquired with a $20 \times$ objective and the positive staining was quantified manually using Image-Pro-Plus 6.0 software $^{60}$.

The TUNEL assay was performed on paraffin-embedded sections of distal femur growth plates using an in situ Cell Death Detection Kit (Roche Diagnostics) according to the manufacturer's instructions. For quantification, the number of TUNEL-positive cells was counted in a section of the entire distal femur growth plate for each sample.

SA-ק-gal staining assay. The activity of SA- $\beta$-gal in cell cultures was detected according to the reported protocols ${ }^{61}$. Briefly, cultured cells were fixed by $0.5 \%$ glutaraldehyde, washed with PBS, then incubated with $1 \mathrm{mg} / \mathrm{ml} \mathrm{X-gal} \mathrm{in} \mathrm{SA-} \beta$-gal staining solution $(5 \mathrm{mM}$ potassium ferrocyanide, $5 \mathrm{mM}$ potassium ferricyanide, and $2 \mathrm{mM} \mathrm{MgCl}_{2}, \mathrm{pH} \mathrm{6.0)}$ at $37^{\circ} \mathrm{C}$ overnight. For frozen sections, SA- $\beta$-gal activity was determined by using an SA- $\beta$-gal kit (Cell Signaling Technology) according to the manufacturer's instructions.

Cell culture. Primary mouse rib chondrocyte cultures: Rib cages were dissected from E18.5 mouse embryos. The soft and bony tissues surrounding the cartilaginous parts of the rib were removed as much as possible. Rib cages were incubated in pronase $\left(2 \mathrm{mg} / \mathrm{ml}\right.$ in PBS) at $37^{\circ} \mathrm{C}$ for $30 \mathrm{~min}$, followed by incubation in collagenase $\mathrm{D}\left(3 \mathrm{mg} / \mathrm{ml}\right.$ in DMEM without serum) at $37^{\circ} \mathrm{C}$ for $30-60 \mathrm{~min}$ to further remove soft tissue. Then the cleaned rib cartilage pieces were transferred into 1.5$\mathrm{ml}$ centrifuge tubes and incubated in collagenase D digestion solution (DMEM 
with $1.5 \mathrm{mg} / \mathrm{ml}$ collagenase $\mathrm{D}$ and $5 \%$ fetal bovine serum (FBS)) overnight at $37^{\circ} \mathrm{C}$. The dissociated chondrocytes were collected and plated in $\alpha$-MEM medium with $10 \%$ FBS.

BMSC cultures: Bone marrow was flushed out from mouse tibia and femurs and was cultured in $\alpha$-MEM with $10 \%$ FBS. After $48 \mathrm{~h}$, non-adherent cells were removed by three washes in PBS and the adherent cells (i.e., BMSCs) were continuously cultured with medium changes every 2 days. After 7-10 days in culture, BMSCs were subcultured. All experiments were performed using cells at the second or third passage if not specified. To induce differentiation and mineralization, BMSCs were cultured in a mineralization medium (growth medium plus $50 \mu \mathrm{g} / \mathrm{ml}$ ascorbic acid, $1 \mathrm{mM} \beta$-glycerophosphate, and $0.01 \mu \mathrm{M}$ dexamethasone).

CFU-F and CFU-O cultures: The total bone marrow was harvested from mouse femurs and tibias, seeded at $1 \times 10^{6}$ cells/well in 12-well plates, cultured for $48 \mathrm{~h}$, and then washed to remove unattached cells. For the CFU-F (fibroblast) assay, the cells were cultured for another 8 days in normal culture medium ( $\alpha$-MEM, $10 \%$ FBS) and then were stained with crystal violet. For the CFU-O (osteoblast) assay, the cells were cultured for another 8 days in mineralization medium and then stained for alkaline phosphatase (ALP) ${ }^{59}$.

The HEK293 cell line was obtained from ATCC and was cultured in DMEM medium with $10 \% \mathrm{FBS}$.

Western blots. Total protein was extracted from mouse rib cartilage or from cell cultures using RIPA buffer. Samples containing equal amounts of protein were separated by SDS-PAGE gel and subjected to standard western blot procedures. Antibodies against SUMO1, SUMO2/3 were purchased from Sigma; anti-RPA70 was purchased from Santa Cruz; anti-p53, anti-p-p53, anti- $\gamma \mathrm{H} 2 \mathrm{AX}$, anti-TRIM28, and anti- $\alpha$-tubulin antibodies were purchased from Cell Signaling Technology. The original uncropped scans of western blots are shown in Supplementary Figure 20, 21. Detailed information about the antibodies used in this study is provided in Supplementary Table 1

Co-immunoprecipitation. HEK293 cells were transfected with Myc-tagged TRIM28, 3XFlag-SENP6, or 3XFlag-SENP6(C1030A $)^{15}$ plasmids using X-fect transfection reagent (Clontech Laboratories). After culturing for $48 \mathrm{~h}$, cells were lysed in RIPA buffer containing protease inhibitor and NEM ( $N$-ethylmaleimide). Myc (Invitrogen, 1:1000) or Flag (Sigma-Aldrich, 1:1000) antibodies were used to immunoprecipitate TRIM28 and SENP6 or the SENP6 mutant (C1030A), respectively. The precipitates were boiled in Laemmli buffer (Bio-Rad) with $5 \% \beta$-mercaptoethanol and loaded onto a 6\% PAGE-SDS gel for western blot assays.

Quantitative RT-PCR (qRT-PCR). Total RNA was extracted form mouse rib cartilage, calvaria, or cell cultures using TRIzol (Invitrogen), followed by use of a GenElute Mammalian Total RNA kit (Sigma) with on-column DNase digestion. First-strand cDNA was generated from $1 \mu \mathrm{g}$ of RNA using SuperScript III Firststrand Synthesis System (Invitrogen). Real-time PCR was performed using the StepOnePlus system and Fast SYBR Green Master Mix (Invitrogen). The relative gene expression levels were calculated using $2^{-\Delta \Delta C T}$ method $^{62}$. The actin gene was used as an internal expression control. The specific primer sequences used in the study are provided in Supplementary Table 2.

$\gamma$ H2AX foci assays. X-ray irradiations were performed at $225 \mathrm{kVp}$; dosage was $2 \mathrm{~Gy}$ at a rate of $0.4 \mathrm{~Gy} / \mathrm{min}$ hardened with a $4-\mathrm{mm}$ removable copper filter ${ }^{63}$. To minimize variations, radiation exposures were conducted at room temperature with cells covered in $2 \mathrm{ml}$ of warmed culture medium. Pre- and post-irradiation, cells were immediately transferred to a $37^{\circ} \mathrm{C}, 5 \% \mathrm{CO}_{2}$ incubator in the same room. Control experiments were performed following exactly the same irradiation protocol but without energizing the X-ray unit. After irradiation, cells were fixed at different time points for plotting the kinetics of $\gamma$-H2AX induction and clearance. Cells were fixed in ice cold $4 \%$ paraformaldehyde for $15 \mathrm{~min}$ at room temperature, permeabilized with $0.5 \%$ Triton X-100:PBS, blocked in NSB buffer (Upstate), and stained with anti- $\gamma-\mathrm{H} 2 \mathrm{AX}$ antibody (Upstate) and anti-mouse AlexaFluor488 secondary antibody (Molecular Probes). Mounting medium containing DAPI was used to counterstain nuclei. Images were acquired with a $100 \times$ objective. The $\gamma$-H2AX foci were scored manually using Image-Pro-Plus 6.0 software, and the average number of foci per cell was calculated from a minimum of 200 cells per time point. Experimental data represent the average of two independent experiments.

RNA sequencing and data analysis. Total RNA was extracted from the primary chondrocytes of Dermo1-Cre;Senp6 ${ }^{f / f}$ and Senp 6 f/f mice rib cartilage using GenElute Mammalian Total RNA Miniprep Kit (Sigma) according to the manufacturer's instructions. RNA was then sequenced by the BGI RNA-seq service ( $n=$ 2). GO analysis was performed using DAVID (http://david.abcc.ncifcrf.gov/). Gene expression clustering was analyzed using Cluster 3.0 and visualized using Java TreeView.

For Gene Set Enrichment Analysis, we used GSEA v2.2.0 on various functional and/or characteristic gene signatures. Gene sets were obtained from the MSigDB database v3.0. Statistical significance was assessed by comparing the enrichment score to enrichment results generated from 1000 random permutations of the gene set to obtain $p$ values (nominal $p$ value).

LC/MS/MS. Sumoylated proteins were enriched from the primary chondrocytes using a SUMO-QAPTURE-T Kit (Enzo-Lifesciences) following the manufacturer's protocol and were analyzed at the Mass Spectrometry Core Facility of Michigan State University. Briefly, samples were purified and concentrated in a onedimensional (1D) SDS-PAGE gel, and the gel bands were dehydrated and then digested using sequencing grade-modified trypsin. Peptides were extracted from the gel by water bath sonication and then re-suspended. Eluted peptides were sprayed into a ThermoFisher Q-Exactive mass spectrometer using a FlexSpray spray ion source. Survey scans were taken in the Orbi trap $(70,000$ resolution, determined at $\mathrm{m} / \mathrm{z} 200$ ) and the top 10 ions in each survey scan were then subjected to automatic higher energy collision-induced dissociation (HCD) with fragment spectra acquired at 17,500 resolution. The resulting MS/MS spectra were converted to peak lists using Mascot Distiller, v2.5.1, and searched against all mouse protein entries available from the UniProt database appended with common laboratory contaminants and from the GPM, using the Mascot searching algorithm, v2.5. The Mascot output was then analyzed using Scaffold v4.4.6 to probabilistically validate protein identifications. Assignments validated using the Scaffold 1\% FDR confidence filter was considered true. After filtering non-specific hits, proteins having at least two peptides detected by MS and more than a 2.2-fold increase in the Dermo1-Cre;Senp 6 fff chondrocytes relative to controls were considered candidate substrates of SENP6.

p53 reporter assay. HEK293 cells (ATCC) were transfected with PG13-Luc (with p53 binding cis-element, $0.5 \mu \mathrm{g})$ and Myc-TRIM28, TRIM28 $2554 \mathrm{R}$, TRIM $28^{\mathrm{K} 779 \mathrm{R}}$, TRIM28 ${ }^{\mathrm{K} 804 \mathrm{R}}$, TRIM28 ${ }^{3 \mathrm{KR}}(0.5 \mu \mathrm{g})$ with HA-SUMO3 $(0.5 \mu \mathrm{g})$ or Flag-SENP6 $(1.5$ $\mu \mathrm{g})$ present or absent based on the experimental strategy. Each transfection included Flag-p53 $(0.05 \mu \mathrm{g})$ and pRL-Renilla $(0.05 \mu \mathrm{g}$, as control). After incubation for $48 \mathrm{~h}$ at $37^{\circ} \mathrm{C}$, the cell lysate was collected for luciferase assay using a DualLuciferase kit (Promega). The firefly-luminescence results were normalized to the Renilla luminescence for internal control of transfection efficiency.

Statistical analysis. All statistical results are presented as the mean \pm standard deviation (S.D.). The differences between two groups were calculated using the two-tailed, unpaired Student's $t$-test; $p$ values $<0.05$ were considered statistically significant. If not specified in the figure legends, the number of animals per group was $n \geq 3$ for the in vivo experiments, including macroscopic phenotyping, skeletal staining, histology, immunohistochemistry, genetic rescues, qRT-PCR, and western blots. The representative images were those in good agreement with the consistent observations. For the in vitro or ex vivo studies, the number of biological replicates $(n)$ is indicated in each figure legend.

Data availability. RNA-seq data that support the findings of this study have been deposited in Gene Expression Omnibus with the accession codes (GSE102856). All relevant data are available from the authors upon reasonable request.

Received: 27 March 2017 Accepted: 29 November 2017 Published online: 10 January 2018

\section{References}

1. Hall B. K. Bones and Cartilage: Developmental and Evolutionary Skeletal Biology (Elsevier Science, 2014)

2. Knothe Tate, M. L., Falls, T. D., McBride, S. H., Atit, R. \& Knothe, U. R. Mechanical modulation of osteochondroprogenitor cell fate. Int. J. Biochem. Cell Biol. 40, 2720-2738 (2008)

3. Lopez-Otin, C., Blasco, M. A., Partridge, L., Serrano, M. \& Kroemer, G. The hallmarks of aging. Cell 153, 1194-1217 (2013)

4. Tahmasebi, S., Ghorbani, M., Savage, P., Gocevski, G. \& Yang, X. J. The SUMO conjugating enzyme Ubc9 is required for inducing and maintaining stem cell pluripotency. Stem Cells 32, 1012-1020 (2014).

5. Qi, Y., Zuo, Y., Yeh, E. T. \& Cheng, J. An essential role of small ubiquitin-like modifier (SUMO)-specific Protease 2 in myostatin expression and myogenesis. J. Biol. Chem. 289, 3288-3293 (2014).

6. Chiu, S. Y., Asai, N., Costantini, F. \& Hsu, W. SUMO-specific protease 2 is essential for modulating p53-Mdm2 in development of trophoblast stem cell niches and lineages. PLoS Biol. 6, e310 (2008).

7. $\mathrm{Li}, \mathrm{T}$. et al. Expression of SUMO-2/3 induced senescence through p53- and pRB-mediated pathways. J. Biol. Chem. 281, 36221-36227 (2006).

8. Bischof, O. et al. The E3 SUMO ligase PIASy is a regulator of cellular senescence and apoptosis. Mol. Cell 22, 783-794 (2006). 
9. Yates, K. E., Korbel, G. A., Shtutman, M., Roninson, I. B. \& DiMaio, D. Repression of the SUMO-specific protease Senp1 induces p53-dependent premature senescence in normal human fibroblasts. Aging Cell 7, 609-621 (2008).

10. Zhang, L., Li, F., Dimayuga, E., Craddock, J. \& Keller, J. N. Effects of aging and dietary restriction on ubiquitination, sumoylation, and the proteasome in the spleen. FEBS Lett. 581, 5543-5547 (2007).

11. Yukita, A. et al. Ubc9 negatively regulates BMP-mediated osteoblastic differentiation in cultured cells. Bone 50, 1092-1099 (2012).

12. Nayak, A., Viale-Bouroncle, S., Morsczeck, C. \& Muller, S. The SUMO-specific isopeptidase SENP3 regulates MLL1/MLL2 methyltransferase complexes and controls osteogenic differentiation. Mol. Cell 55, 47-58 (2014).

13. arc, O. C. et al. Identification of new susceptibility loci for osteoarthritis (arcOGEN): a genome-wide association study. Lancet 380, 815-823 (2012).

14. Johnson, K., Reynard, L. N. \& Loughlin, J. Functional characterisation of the osteoarthritis susceptibility locus at chromosome 6q14.1 marked by the polymorphism rs9350591. BMC Med. Genet. 16, 81 (2015).

15. Dou, H., Huang, C., Singh, M., Carpenter, P. B. \& Yeh, E. T. Regulation of DNA repair through deSUMOylation and SUMOylation of replication protein A complex. Mol. Cell 39, 333-345 (2010).

16. Gibbs-Seymour, I. et al. Ubiquitin-SUMO circuitry controls activated fanconi anemia ID complex dosage in response to DNA damage. Mol. Cell 57, 150-164 (2015).

17. Mukhopadhyay, D., Arnaoutov, A. \& Dasso, M. The SUMO protease SENP6 is essential for inner kinetochore assembly. J. Cell. Biol. 188, 681-692 (2010).

18. Liu, X., Chen, W., Wang, Q., Li, L. \& Wang, C. Negative regulation of TLR inflammatory signaling by the SUMO-deconjugating enzyme SENP6. PLoS Pathog. 9, e1003480 (2013).

19. Chen, D. et al. A microarray analysis of the emergence of embryonic definitive hematopoiesis. Exp. Hematol. 35, 1344-1357 (2007)

20. Ruzankina, Y. et al. Deletion of the developmentally essential gene ATR in adult mice leads to age-related phenotypes and stem cell loss. Cell Stem Cell $\mathbf{1}$, 113-126 (2007).

21. Park, D. et al. Endogenous bone marrow MSCs are dynamic, fate-restricted participants in bone maintenance and regeneration. Cell Stem Cell 10, 259-272 (2012).

22. Logan, M. et al. Expression of Cre Recombinase in the developing mouse limb bud driven by a Prxl enhancer. Genesis 33, 77-80 (2002).

23. Muzumdar, M. D., Tasic, B., Miyamichi, K., Li, L. \& Luo, L. A global doublefluorescent Cre reporter mouse. Genesis 45, 593-605 (2007).

24. Morini, S., Continenza, M. A., Ricciardi, G., Gaudio, E. \& Pannarale, L. Development of the microcirculation of the secondary ossification center in rat humeral head. Anat. Rec. A Discov. Mol. Cell. Evol. Biol. 278, 419-427 (2004).

25. Mukhopadhyay, D. et al. SUSP1 antagonizes formation of highly SUMO2/3conjugated species. J. Cell. Biol. 174, 939-949 (2006).

26. Donehower, L. A. \& Lozano, G. 20 years studying p53 functions in genetically engineered mice. Nat. Rev. Cancer 9, 831-841 (2009).

27. Chen, L. \& Chen, J. MDM2-ARF complex regulates p53 sumoylation. Oncogene 22, 5348-5357 (2003).

28. Santiago, A., Li, D., Zhao, L. Y., Godsey, A. \& Liao, D. p53 SUMOylation promotes its nuclear export by facilitating its release from the nuclear export receptor CRM1. Mol. Biol. Cell 24, 2739-2752 (2013).

29. Rodriguez, M. S. et al. SUMO-1 modification activates the transcriptional response of p53. EMBO J. 18, 6455-6461 (1999).

30. Cheng, C. T., Kuo, C. Y. \& Ann, D. K. KAPtain in charge of multiple missions: emerging roles of KAP1. World J. Biol. Chem. 5, 308-320 (2014).

31. Wang, C. et al. MDM2 interaction with nuclear corepressor KAP1 contributes to p53 inactivation. EMBO J. 24, 3279-3290 (2005).

32. Mascle, X. H., Germain-Desprez, D., Huynh, P., Estephan, P. \& Aubry, M. Sumoylation of the transcriptional intermediary factor 1beta (TIF1beta), the Co-repressor of the KRAB Multifinger proteins, is required for its transcriptional activity and is modulated by the KRAB domain. J. Biol. Chem. 282, 10190-10202 (2007).

33. Ivanov, A. V. et al. PHD domain-mediated E3 ligase activity directs intramolecular sumoylation of an adjacent bromodomain required for gene silencing. Mol. Cell 28, 823-837 (2007).

34. Lujambio, A. et al. Non-cell-autonomous tumor suppression by p53. Cell 153, 449-460 (2013)

35. Sarge, K. D. \& Park-Sarge, O. K. Sumoylation and human disease pathogenesis. Trends Biochem. Sci. 34, 200-205 (2009).

36. Rufini, A., Tucci, P., Celardo, I. \& Melino, G. Senescence and aging: the critical roles of p53. Oncogene 32, 5129-5143 (2013).

37. Liu, D. et al. Puma is required for p53-induced depletion of adult stem cells Nat. Cell Biol. 12, 993-998 (2010).

38. Lengner, C. J. et al. Osteoblast differentiation and skeletal development are regulated by Mdm2-p53 signaling. J. Cell Biol. 172, 909-921 (2006).

39. Doyle, J. M., Gao, J., Wang, J., Yang, M. \& Potts, P. R. MAGE-RING protein complexes comprise a family of E3 ubiquitin ligases. Mol. Cell 39, 963-974 (2010).
40. Yang, B. et al. MAGE-A, mMage-b, and MAGE-C proteins form complexes with KAP1 and suppress p53-dependent apoptosis in MAGE-positive cell lines. Cancer Res. 67, 9954-9962 (2007).

41. Deschenes-Simard, X. et al. Tumor suppressor activity of the ERK/MAPK pathway by promoting selective protein degradation. Genes Dev. 27, 900-915 (2013).

42. Kuo, C. Y. et al. An arginine-rich motif of ring finger protein 4 (RNF4) oversees the recruitment and degradation of the phosphorylated and SUMOylated Kruppel-associated box domain-associated protein 1 (KAP1)/TRIM28 protein during genotoxic stress. J. Biol. Chem. 289, 20757-20772 (2014).

43. Li, X. et al. Role for KAP1 serine 824 phosphorylation and sumoylation/ desumoylation switch in regulating KAP1-mediated transcriptional repression. J. Biol. Chem. 282, 36177-36189 (2007).

44. Lee, Y. K., Thomas, S. N., Yang, A. J. \& Ann, D. K. Doxorubicin down-regulates Kruppel-associated box domain-associated protein 1 sumoylation that relieves its transcription repression on p21WAF1/CIP1 in breast cancer MCF-7 cells. J. Biol. Chem. 282, 1595-1606 (2007).

45. Cheng, Z. et al. Functional characterization of TIP60 sumoylation in UVirradiated DNA damage response. Oncogene 27, 931-941 (2008).

46. Hattersley, N., Shen, L., Jaffray, E. G. \& Hay, R. T. The SUMO protease SENP6 is a direct regulator of PML nuclear bodies. Mol. Biol. Cell 22, 78-90 (2011).

47. Williams, A. B. \& Schumacher, B. p53 in the DNA-Damage-Repair Process. Cold Spring Harb. Perspect. Med. https://doi.org/10.1101/cshperspect.a026070 a026070 (2016).

48. Cao, L., Li, W., Kim, S., Brodie, S. G. \& Deng, C. X. Senescence, aging, and malignant transformation mediated by p53 in mice lacking the Brcal fulllength isoform. Genes Dev. 17, 201-213 (2003).

49. Lim, D. S. et al. Analysis of ku80-mutant mice and cells with deficient levels of p53. Mol. Cell. Biol. 20, 3772-3780 (2000).

50. Barde, I. et al. Regulation of episomal gene expression by KRAB/KAP1mediated histone modifications. J. Virol. 83, 5574-5580 (2009).

51. Turelli, P. et al. Interplay of TRIM 28 and DNA methylation in controlling human endogenous retroelements. Genome Res. 24, 1260-1270 (2014).

52. Yang, B. X. et al. Systematic identification of factors for provirus silencing in embryonic stem cells. Cell 163, 230-245 (2015).

53. Garvin, A. J. et al. The deSUMOylase SENP7 promotes chromatin relaxation for homologous recombination DNA repair. EMBO Rep. 14, 975-983 (2013).

54. Jin, Z. L., Pei, H., Xu, Y. H., Yu, J. \& Deng, T. The SUMO-specific protease SENP5 controls DNA damage response and promotes tumorigenesis in hepatocellular carcinoma. Eur. Rev. Med. Pharmacol. Sci. 20, 3566-3573 (2016).

55. Zunino, R., Schauss, A., Rippstein, P., Andrade-Navarro, M. \& McBride, H. M. The SUMO protease SENP5 is required to maintain mitochondrial morphology and function. J. Cell Sci. 120, 1178-1188 (2007).

56. Chen, H. et al. Apelin inhibits the development of diabetic nephropathy by regulating histone acetylation in Akita mouse. J. Physiol. 592, 505-521 (2014).

57. Li, J. et al. Sclt1 deficiency causes cystic kidney by activating ERK and STAT3 signaling. Hum. Mol. Genet. 26, 2949-2960 (2017).

58. Burgers, T. A. et al. Mice lacking pten in osteoblasts have improved intramembranous and late endochondral fracture healing. PLOS ONE 8, e63857 (2013).

59. Yang, T. et al. E-selectin ligand 1 regulates bone remodeling by limiting bioactive TGF-beta in the bone microenvironment. Proc. Natl Acad. Sci. USA 110, 7336-7341 (2013)

60. Yang, T. et al. E-selectin ligand-1 regulates growth plate homeostasis in mice by inhibiting the intracellular processing and secretion of mature TGF-beta. J. Clin. Invest. 120, 2474-2485 (2010).

61. Debacq-Chainiaux, F., Erusalimsky, J. D., Campisi, J. \& Toussaint, O. Protocols to detect senescence-associated beta-galactosidase (SA-betagal) activity, a biomarker of senescent cells in culture and in vivo. Nat. Protoc. 4, 1798-1806 (2009).

62. Huang, J. et al. Histone acetyltransferase PCAF regulates inflammatory molecules in the development of renal injury. Epigenetics 10, 62-72 (2015).

63. Mariotti, L. G. et al. Use of the gamma-H2AX assay to investigate DNA repair dynamics following multiple radiation exposures. PLoS ONE 8, e79541 (2013).

\section{Acknowledgements}

We thank the members of the Yang laboratory and other members of the Program for Skeletal Disease and Tumor Metastasis at Van Andel Research Institute for discussion; Drs. Gerd Pfeifer and Piroska Szabó for suggestion; the Vivarium and Transgenics Core for animal technical assistance; the Small-Animal Imaging Core for X-ray irradiation assay assistance; and David Nadziejka for technical editing of this manuscript. This work was supported by VARI startup funds (T.Y.) and Arthritis National Research Foundation (30 40379) (T.Y.), and partially by Chinese 111 Project Grants B06018 and B16036 (L.Z.).

\section{Author contributions}

Jianshuang, L., L.Z., and T.Y. designed the research studies. Jianshuang, L, D.L., H.D., H. L., K.W., W.W., L.Z., and T.Y. conducted the experiments, acquired the data, and 
analyzed the results. H.D., Jiada.L., E.T.H.Y., and B.O.W. provided reagents, technical support, and discussions. Jianshuang, L., L.Z., and T.Y. wrote the manuscript.

\section{Additional information}

Supplementary Information accompanies this paper at https://doi.org/10.1038/s41467017-02413-3.

Competing interests: The authors declare no competing financial interests.

Reprints and permission information is available online at http://npg.nature.com/ reprintsandpermissions/

Publisher's note: Springer Nature remains neutral with regard to jurisdictional claims in published maps and institutional affiliations. (c) (i) Open Access This article is licensed under a Creative Commons Attribution 4.0 International License, which permits use, sharing, adaptation, distribution and reproduction in any medium or format, as long as you give appropriate credit to the original author(s) and the source, provide a link to the Creative Commons license, and indicate if changes were made. The images or other third party material in this article are included in the article's Creative Commons license, unless indicated otherwise in a credit line to the material. If material is not included in the article's Creative Commons license and your intended use is not permitted by statutory regulation or exceeds the permitted use, you will need to obtain permission directly from the copyright holder. To view a copy of this license, visit http://creativecommons.org/ licenses/by/4.0/.

(C) The Author(s) 2018 